\title{
Eigenvalue Approximation by Mixed and Hybrid Methods
}

\author{
By B. Mercier, J. Osborn*, J. Rappaz** and P. A. Raviart
}

\begin{abstract}
Rate of convergence estimates are derived for the approximation of eigenvalues and eigenvectors by mixed and hybrid methods. Several closely related abstract results on spectral approximation are proved. These results are then applied to a variety of finite element methods of mixed and hybrid type: a mixed method for 2 nd order problems, mixed methods for 4 th order problems, a hybrid method for 2 nd order problems, and two mixed methods for the Stokes eigenvalue problem.
\end{abstract}

1. Introduction. The use of mixed and hybrid methods for the approximate solution of source problems has received considerable attention. We mention the works of Herrmann [20], [21], Glowinski [19], Miyoshi [29], Oden [33], Johnson [25], Mercier [27], Ciarlet-Raviart [10], Brezzi [6], [7], Scholz [42], [43], BrezziRaviart [8], Oden-Reddy [35], Raviart-Thomas [40], [41], Falk [14], Falk-Osborn [15], Rannacher [39], and Babuška-Osborn-Pitkäranta [5].

Nemat-Nasser [30], [31], [32] has observed that mixed methods are effective for the approximation of eigenvalues of differential equations with rough coefficients. Babuška-Osborn [3] establish rate of convergence estimates for these methods as they pertain to ordinary differential equations.

Canuto [9] and Ishihara [23], [24] have studied eigenvalue approximations for the biharmonic problem by mixed methods. For the 2 nd order problems, Mercier-Rappaz [28] derived optimal estimates for a hybrid method, and Ishihara [22] obtained estimates for a mixed method.

It is the purpose of this paper to prove several closely related abstract results on eigenvalue approximation that can be applied to a wide variety of finite element eigenvalue approximation methods of mixed or hybrid type (including most of those mentioned above).

In Sections 2-6, we prove the abstract results. These are obtained as a consequence of results of Osborn [36] and Descloux-Nassif-Rappaz [12], [13]. In Section 7, we apply these results to several finite element methods of mixed or hybrid type: a mixed method for the 2 nd order elliptic equations, mixed methods for 4 th order problems, a hybrid method for 2 nd order problems, and two methods for the approximations of the eigenvalues of the Stokes problem.

Received April 9, 1980; revised August 14, 1980.

1980 Mathematics Subject Classification. Primary 65N15, 65N30, 65N25.

- The work of the second author was partially supported by the National Science Foundation under Grant No. MCS 78-02851 and by the United States Army under Control No. DAAG 29-75-C-0024 and was completed during his visit at the Université Pierre et Marie Curie.

** The work of the third author was supported by the Fonds National Suisse de la Recherche Scientifique. 
We describe now the general types of problems that we will consider. Let $X$ and $W$ be two complex Hilbert spaces with scalar products and norms $(\cdot, \cdot)_{X},\|\cdot\|_{X}$, $(\cdot, \cdot)_{W},\|\cdot\|_{W}$, respectively, and let $a: X \times X \rightarrow \mathbf{C}, b: X \times W \rightarrow \mathbf{C}, r: X \times X \rightarrow$ $\mathrm{C}$, and $S: W \times W \rightarrow \mathrm{C}$ be continuous sesquilinear forms. We consider eigenvalue problems of the following two forms:

Find $\lambda \in \mathbf{C}, 0 \neq(u, p) \in X \times W$ satisfying

$$
\begin{cases}a(u, v)+\overline{b(v, p)}=\lambda r(u, v), & \forall v \in X, \\ b(u, q)=0, & \forall q \in W .\end{cases}
$$

Find $\lambda \in \mathbf{C}, 0 \neq(u, p) \in X \times W$ satisfying

$$
\begin{cases}a(u, v)+\overline{b(v, p)}=0, & \forall v \in X, \\ b(u, q)=-\lambda s(p, q), & \forall q \in W .\end{cases}
$$

We are interested in the approximations of eigenvalues of (Q1) and (Q2), and toward this end we suppose we are given families of finite-dimensional spaces $X_{h} \subset X$ and $W_{h} \subset W$ and consider the following approximate eigenvalue problems:

Find $\lambda_{h} \in \mathbf{C}, 0 \neq\left(u_{h}, p_{h}\right) \in X_{h} \times W_{h}$ satisfying

$(\mathbf{Q} 1)_{h}$

$$
\begin{cases}a\left(u_{h}, v_{h}\right)+\overline{b\left(v_{h}, p_{h}\right)}=\lambda_{h} r\left(u_{h}, v_{h}\right), & \forall v_{h} \in X_{h}, \\ b\left(u_{h}, q_{h}\right)=0, & \forall q_{h} \in W_{h} .\end{cases}
$$

Find $\lambda_{h} \in \mathbf{C}, 0 \neq\left(u_{h}, p_{h}\right) \in X_{h} \times W_{h}$ satisfying

$$
\begin{cases}a\left(u_{h}, v_{h}\right)+\overline{b\left(v_{h}, p_{h}\right)}=0, & \forall v_{h} \in X_{h}, \\ b\left(u_{h}, q_{h}\right)=-\lambda_{h} s\left(p_{h}, q_{h}\right), & \forall q_{h} \in W_{h} .\end{cases}
$$

We now regard $\lambda_{h}, u_{h}$, and $p_{h}$ as approximations to $\lambda, u$, and $p$, respectively, and study the errors in these approximations.

Notations. Throughout this paper we shall use the Sobolev spaces $W^{m p}(\Omega)$, where $\Omega$ is an open set in $\mathbf{R}^{n}, m$ is a nonnegative integer, and $1<p<\infty$, with the usual norms and seminorms $\|\cdot\|_{m,, \Omega}$ and $|\cdot|_{m, p, \Omega}$. When $p=2$, we denote $W^{m, 2}(\Omega)$ by $H^{m}(\Omega)$ and write

$$
|v|_{m, \Omega}=|v|_{m, 2, \Omega}, \quad\|v\|_{m, \Omega}=\|v\|_{m, 2, \Omega} .
$$

We also use the vector versions of these spaces with the usual product norms and notations: $\mathbf{H}^{1}(\Omega)$, for example, will denote the space of functions $u(x)=$ $\left(u_{1}(x), u_{2}(x), \ldots, u_{d}(x)\right)$ with $u_{j} \in H^{1}(\Omega), j=1,2, \ldots, d$; the dimension $d$ will be understood from the context. $H_{0}^{1}(\Omega)$ is the subspace of functions in $H^{1}(\Omega)$ that vanish on $\Gamma=\partial \Omega . H^{1 / 2}(\Gamma)$ is the space of traces $v / \Gamma$ of functions $v \in H^{1}(\Omega)$ and $H^{-1 / 2}(\Gamma)$ is the dual space of $H^{1 / 2}(\Gamma) . H(\operatorname{div}, \Omega)=\left\{q \in \mathbf{L}^{2}(\Omega)=\left(L^{2}(\Omega)\right)^{n}: \operatorname{div} q \in\right.$ $\left.L^{2}(\Omega)\right\}$ where div is the divergence operator.

2. A General Spectral Approximation Result. General results on spectral approximation for compact operators were obtained by Bramble-Osborn [4] and Osborn [36]. Descloux-Nassif-Rappaz [12], [13] have refined and extended some of the results of [4], [36].

In this section we state two general results on the approximation of eigenvalues and eigenvectors of compact operators, referring to [36], [12] for proofs. 
Let $T$ be a compact operator on a complex Banach space $H$ with norm $|\cdot|_{H}$ and let $\left\{T_{h}\right\}_{0<h<1}$ be a family of compact operators on $H$ satisfying

$$
\lim _{h \rightarrow 0}\left|T-T_{h}\right|=0 \text {, }
$$

where $|\cdot|$ is the operator norm on $H$. The spectrum of $T$ consists of a countable set of complex numbers, and each nonzero number in the spectrum is an isolated eigenvalue. Let $\mu$ be a nonzero eigenvalue of $T$. Then there exists a least integer $\alpha$ such that $\operatorname{Ker}\left((\mu-T)^{\alpha}\right)=\operatorname{Ker}\left((\mu-T)^{\alpha+1}\right) \equiv E$, with $\operatorname{dim} E \equiv m<\infty$. $\alpha$ is called the ascent of $(\mu-T)$, the elements of $E$ the generalized eigenvectors of $T$ corresponding to $\mu$, and $m$ the algebraic multiplicity of $\mu$. The order of a generalized eigenvector $f \in E$ is the smallest positive integer $j$ such that $f \in$ $\operatorname{Ker}\left((\mu-T)^{j}\right)$.

Let $T^{*}: H^{*} \rightarrow H^{*}$ be the adjoint operator of $T$ defined on the dual space $H^{*}$ of $H$, i.e. the space of bounded, conjugate linear forms on $H$. Then $\bar{\mu}$ is an eigenvalue of $T^{*}$ with algebraic multiplicity $m$. The ascent of $\left(\bar{\mu}-T^{*}\right)$ will be $\alpha$. Let $E^{*}=\operatorname{Ker}\left(\left(\bar{\mu}-T^{*}\right)^{\alpha}\right)$ be the space of generalized eigenvectors of $T^{*}$ corresponding to $\bar{\mu}$.

It is well known, as a consequence of (2.1), that exactly $m$ eigenvalues of $T_{h}$ (counted according to algebraic multiplicity) converge to $\mu$; we denote these by $\mu_{1 h}, \mu_{2 h}, \ldots, \mu_{m h}$.

THEOREM 2.1. There are constants $C$ and $h_{0}$ such that, for $0<h<h_{0}$,

$$
\begin{aligned}
& \left|\mu-\frac{1}{m} \sum_{i=1}^{m} \mu_{i h}\right|<C\left\{\sup _{\substack{u \in E^{*}|u|_{H}=1 \\
v^{*} \in E,\left|v^{*}\right|_{H^{*}}=1}}\left|\left\langle\left(T-T_{h}\right) u, v^{*}\right\rangle\right|\right. \\
& \left.+\left|\left(T-T_{h}\right)_{/ E}\right| \cdot\left|\left(T^{*}-T_{h}^{*}\right)_{/ E^{*}}\right|_{*}\right\} \equiv C \delta_{h}, \\
& \text { where }\left(T-T_{h}\right)_{/ E} \text { denotes the restriction of } T-T_{h} \text { to } E \text { and }|\cdot|_{*} \text { is the operator }
\end{aligned}
$$
norm on $H^{*}$.

Given two closed subspaces $M$ and $N$ of $H$, we define

$$
\delta(M, N)=\sup _{\substack{u \in M \\|u|_{H}=1}} \inf _{v \in N}|u-v|_{H}
$$

and then define $\hat{\delta}(M, N)$, the gap between $M$ and $N$, by

$$
\hat{\delta}(M, N)=\max [\delta(M, N), \delta(N, M)] \text {. }
$$

Let $E_{h}$ be the direct sum of the generalized eigensubspaces of $T_{h}$ corresponding to $\mu_{1 h}, \mu_{2 h}, \ldots, \mu_{m h}$. As a consequence of $(2.1), \operatorname{dim} E_{h}=\operatorname{dim} E=m$ for small $h$ and the eigenvector error, as measured by $\hat{\delta}\left(E, E_{h}\right)$, is estimated by

THeOREM 2.2. There is a constant $C$ such that

$$
\hat{\delta}\left(E, E_{h}\right)<C\left|\left(T-T_{h}\right)_{/ E}\right| .
$$


Theorems 2.1 and 2.2 have been used to analyze a wide variety of eigenvalue problems; cf. [12], [13], [36].

3. A General Result on Variationally Posed Eigenvalue Problems. We consider in this section the approximation of variationally posed eigenvalue problems, i.e., eigenvalue problems of the form

$$
\left\{\begin{array}{l}
\text { Find } \lambda \in \mathbf{C}, 0 \neq U \in H_{1} \text { satisfying } \\
A(U, V)=\lambda B(U, V), \quad \forall V \in H_{2},
\end{array}\right.
$$

where $H_{1}$ and $H_{2}$ are complex Hilbert spaces with norms $\|\cdot\|_{1}$ and $\|\cdot\|_{2}$ and $A$ : $H_{1} \times H_{2} \rightarrow \mathrm{C}$ and $B: H_{1} \times H_{2} \rightarrow \mathrm{C}$ are continuous sesquilinear forms satisfying

$$
\begin{gathered}
\inf _{\substack{U \in H_{1} \\
\|U\|_{1}=1}} \sup _{\substack{V \in H_{2} \\
\|V\|_{2}=1}}|A(U, V)|=\alpha_{1}>0, \\
\sup _{U \in H_{1}}|A(U, V)|>0 \quad \text { for all } 0 \neq V \in H_{2},
\end{gathered}
$$

and

$$
T: H_{1} \rightarrow H_{1} \text { is compact, }
$$

where $T$ satisfies

$$
A(T U, V)=B(U, V), \quad \forall V \in H_{2} \text {. }
$$

We are interested in approximating the eigenvalues of (3.1), and toward this end we suppose we are given families of finite-dimensional subspaces $S_{1 h} \subset H_{1}$ and $S_{2 h} \subset H_{2}, 0<h<1$, with $\operatorname{dim} S_{1 h}=\operatorname{dim} S_{2 h}$, and we consider the approximate eigenvalue problem

$$
\left\{\begin{array}{l}
\text { Find } \lambda_{h} \in \mathbf{C}, 0 \neq U_{h} \in S_{1 h} \text { satisfying } \\
A\left(U_{h}, V_{h}\right)=\lambda_{h} B\left(U_{h}, V_{h}\right), \quad \forall V_{h} \in S_{2 h}
\end{array}\right.
$$

Concerning (3.5) we assume

$$
\inf _{\substack{U_{h} \in S_{1 h} \\\left\|U_{h}\right\|_{1}=1}} \sup _{\substack{V_{h} \in S_{2 h} \\\left\|V_{h}\right\|_{2}=1}}\left|A\left(U_{h}, V_{h}\right)\right|>\alpha_{2}>0,
$$

where $\alpha_{2}$ is independent of $h$, and

$$
\lim _{h \rightarrow 0} \inf _{U_{h} \in S_{1 h}}\left\|U-U_{h}\right\|_{1}=0 \text { for each } U \in H_{1} .
$$

$\lambda, U$ is an eigenpair of (3.1) if and only if $\lambda T U=U, U \neq 0$, i.e., if and only if $\mu=1 / \lambda, U$ is an eigenpair of $T$. We define the algebraic multiplicity of $\lambda$ as the algebraic multiplicity of $\mu$ as an eigenvaue of $T$. The generalized eigensubspace $E=\operatorname{Ker}\left((\mu-T)^{\alpha}\right)$, where $\alpha$ is the ascent of $(\mu-T)$, can be characterized in terms of the form $A$ and $B$ as follows. For an integer $j>1$, a nonzero vector $U^{j}$ is a generalized eigenvector of order $j$ if

$$
A\left(U^{j}, V\right)=\lambda B\left(U^{j}, V\right)+\lambda A\left(U^{j-1}, V\right), \quad \forall V \in H_{2},
$$

for some nonzero generalized eigenvector $U^{j-1}$ of order $j-1$.

If $\lambda$ is an eigenvalue of (3.1) then $\lambda$ will have adjoint eigenvectors $V$, i.e., nonzero $V \in H_{2}$ satisfying

$$
A(U, V)=\lambda B(U, V), \quad \forall U \in H_{1}
$$


(3.8) holds if and only if $\bar{\lambda} T_{*} V=V$, where $T_{*}: H_{2} \rightarrow H_{2}$ satisfies

$$
A(T U, V)=A\left(U, T_{*} V\right), \quad \forall U \in H_{1}, V \in H_{2} .
$$

$T_{*}$ is formally the adjoint of $T$ with respect to the form $A$. The ascent of $\left(\bar{\mu}-T_{*}\right)$ is the same as the ascent of $(\mu-T)$. Denote the generalized adjoint eigenspace (i.e. the generalized eigenspace corresponding to $\bar{\mu}$ and $T_{*}$ ) by $E_{*}=$ $\operatorname{Ker}\left(\left(\bar{\mu}-T_{*}\right)^{\alpha}\right) \cdot V^{j}$ is an adjoint generalized eigenvector of order $j$ if

$$
A\left(U, V^{j}\right)=\lambda B\left(U, V^{j}\right)+\lambda A\left(U, V^{j-1}\right), \quad \forall U \in H_{1},
$$

for some adjoint generalized eigenvector $V^{j-1}$ of order $j-1$.

Let $\lambda$ be an eigenvalue of (3.1) and let $m$ be its algebraic multiplicity. As $h$ tends to zero, exactly $m$ eigenvalues $\lambda_{1 h}, \lambda_{2 h}, \ldots, \lambda_{m h}$ of (3.5) (counted according to algebraic multiplicity) converge to $\lambda$. Let $\hat{\lambda}_{h}=(1 / m) \sum_{i=1}^{m} \lambda_{i h}$ and let $E_{h}$ be the direct sum of the generalized eigenspaces corresponding to $\lambda_{1 h}, \lambda_{2 h}, \ldots, \lambda_{m h}$. Let

$$
\varepsilon_{h}=\sup _{\substack{U \in E \\\|U\|_{1}=1}} \inf _{x \in S_{1 h}}\|U-\chi\|_{1} \text { and } \varepsilon_{h}^{*}=\sup _{\substack{V \in E^{*} \\\|V\|_{2}=1}} \inf _{\eta \in S_{2 h}}\|V-\eta\|_{2} .
$$

We are now ready to state our fundamental error estimate.

THEOREM 3.1. There are constants $C$ and $h_{0}>0$ such that, for $0<h<h_{0}$,

$$
\begin{gathered}
\left|\lambda-\hat{\lambda}_{h}\right|<C \varepsilon_{h} \varepsilon_{h}^{*}, \\
\left|\lambda-\lambda_{j h}\right|<C\left(\varepsilon_{h} \varepsilon_{h}^{*}\right)^{1 / \alpha}, \quad j=1,2, \ldots, m,
\end{gathered}
$$

and

$$
\hat{\delta}\left(E, E_{h}\right)<C \varepsilon_{h} .
$$

For a proof of this theorem, in the case when the ascent is one, we refer to Babuška and Aziz [2] and Fix [16]. For a complete discussion of this theorem and a proof in the general case (which is based on Theorem 2.1), we refer to Kolata [26].

We now turn to the application of this result to a certain class of eigenvalue problems of type (Q1) and (Q2). Let $a$ and $b$ be continuous sesquilinear forms on $X \times X$ and $X \times W$, respectively, and assume

$$
\operatorname{Re} a(u, u) \geqslant \beta_{1}\|u\|_{X}^{2}, \quad \forall u \in V, \beta_{1}>0,
$$

where $V=\{v \in X: b(v, q)=0, \forall q \in W\}$, and

$$
\sup _{u \in X} \frac{|b(u, q)|}{\|u\|_{X}} \geqslant \gamma_{1}\|q\|_{W}, \quad \forall q \in W, \gamma_{1}>0 .
$$

Let $X_{h} \subset X$ and $W_{h} \subset W$ be finite-dimensional spaces and assume

$$
\operatorname{Re} a\left(u_{h}, u_{h}\right) \geqslant \beta_{2}\left\|u_{h}\right\|_{X}^{2}, \quad \forall u_{h} \in V_{h},
$$

where $V_{h}=\left\{v_{h} \in X_{h}: b\left(v_{h}, q_{h}\right)=0, \forall q_{h} \in W_{h}\right\}, \beta_{2}$ independent of $h$,

$$
\sup _{u_{h} \in X_{h}} \frac{\left|b\left(u_{h}, q_{h}\right)\right|}{\left\|u_{h}\right\|_{X}} \geqslant \gamma_{2}\left\|q_{h}\right\|_{W}, \quad \forall q_{h} \in W_{h},
$$

$\gamma_{2}$ independent of $h$, and

$$
\lim _{h \rightarrow 0} \inf _{\left(u_{h}, q_{h}\right) \in X_{h} \times W_{h}}\left(\left\|u-u_{h}\right\|_{X}+\left\|q-q_{h}\right\|_{W}\right)=0,
$$

for each $(u, q) \in X \times W$. 
We then consider the eigenvalue problems of type (Q1) and (Q2) with these hypotheses satisfied. These problems and the associated finite-dimensional problems are easily seen to be of the form (3.1) and (3.5), respectively, with the following identifications:

$$
\begin{aligned}
& H_{1}=H_{2}=X \times W, \\
& U=(u, p), \\
& V=(v, q), \\
& A(U, V)=A((u, p),(v, q))=a(u, v)+\overline{b(v, p)}+b(u, q), \\
& B(U, V)=B((u, p),(v, q))= \begin{cases}r(u, v) & \text { for problem }(\mathrm{Q} 1), \\
-s(p, q) & \text { for problem (Q2), }\end{cases} \\
& S_{1 h}=S_{2 h}=X_{h} \times W_{h} .
\end{aligned}
$$

It is well known (Brezzi [6], Babuška [1]) that conditions (3.12)-(3.16) ensure the validity of (3.2), (3.3), (3.6), and (3.7). In addition, we assume (3.4) holds with $A$ and $B$ defined as above.

Thus, all of the hypotheses concerning (3.1) and (3.5), with the above identifications, are satisfied, and the estimates of Theorem 3.1 hold. We will write out these estimates in a special case. Suppose the forms $a, r$, and $s$ are all positive definite. Then $A$ and $B$ are hermitian symmetric, the eigenvalues $\lambda$ are all positive, and all generalized eigenvectors are eigenvectors (we have $\alpha=1$, and $m$ is the geometric multiplicity of $\lambda$ ). The estimates of Theorem 3.1 thus have the form

$$
\begin{gathered}
\left|\lambda-\lambda_{i h}\right| \leqslant C \varepsilon_{h}^{2}, \quad i=1,2, \ldots, m, \\
\hat{\delta}\left(E, E_{h}\right) \leqslant C \varepsilon_{h},
\end{gathered}
$$

where $E$ is the eigenspace corresponding to $\lambda, E_{h}$ is the direct sum of the eigenspaces corresponding to $\lambda_{1 h}, \lambda_{2 h}, \ldots, \lambda_{m h}$, and

$$
\varepsilon_{h}=\sup _{\substack{(u, p) \in E \\\|u\|_{X}+\|p\|_{W}=1}} \inf _{\left(v_{h}, q_{h}\right) \in X_{h} \times W_{h}}\left(\left\|u-v_{h}\right\|_{X}+\left\|p-q_{h}\right\|_{W}\right) .
$$

We refer to problems with the formal structure of $(Q 1)$ or (Q2), which satisfy (3.12)-(3.16) and (3.4), as problems satisfying the full Brezzi hypotheses. There are, however, other problems of type (Q1) or (Q2) which do not satisfy the Brezzi hypotheses (in terms of the usual norms that have been used in their analysis).

In general, in the case of problems of type (Q1), (3.12) holds but not (3.13), whereas, in the case of problems of type (Q2), (3.13) holds but not (3.12). In both cases the operator $T$ is not defined and that is the main reason why we cannot always apply the results of Section 3.

We now turn to the consideration of these problems.

4. A Result on a Nonconforming Approximation Method. We analyze in this section a class of nonconforming approximations to variationally posed eigenvalue problems.

Let $X \subset H$ be two complex Hilbert spaces with scalar products $((\cdot, \cdot)),(\cdot, \cdot)$ and norms $\|\cdot\|,|\cdot|$, respectively. We suppose the injection of $X$ into $H$ is continuous, but not necessarily compact. Let $V$ be a closed subspace of $X$ and let 
$a(\cdot, \cdot)$ and $r(\cdot, \cdot)$ be bounded, sesquilinear forms on $X \times X$ and $H \times H$, respectively. We then consider the eigenvalue problem

$$
\left\{\begin{array}{l}
\text { Find } \lambda \in \mathbf{C}, 0 \neq u \in V \text { satisfying } \\
a(u, v)=\lambda r(u, v), \quad \forall v \in V .
\end{array}\right.
$$

Next, we suppose we are given a family $\left\{V_{h}\right\}_{0<h<1}$ of finite-dimensional subspaces of $X$ and consider the approximate problems

$$
\left\{\begin{array}{l}
\text { Find } \lambda_{h} \in \mathbf{C}, 0 \neq u_{h} \in V_{h} \text { satisfying } \\
a\left(u_{h}, v_{h}\right)=\lambda_{h} r\left(u_{h}, v_{h}\right), \quad \forall v_{h} \in V_{h} .
\end{array}\right.
$$

(4.1) is a variationally formulated eigenvalue problem and, since $V_{h} \not \subset V$ in general, (4.2) represents a nonconforming approximation to (4.1).

Regarding the form $a(\cdot, \cdot)$ we further assume

$$
\begin{gathered}
\operatorname{Re} a(u, u) \geqslant \alpha\|u\|^{2}, \quad \forall u \in V, \\
\operatorname{Re} a\left(u_{h}, u_{h}\right)>0, \quad \forall u_{h} \in V_{h}, u_{h} \neq 0 \text { and } \forall h .
\end{gathered}
$$

In order to analyze this approximation method we introduce the bounded operators $T, T_{*}, T_{h}, T_{* h}: H \rightarrow H$ defined by:

For $f \in H$

$$
\begin{array}{cl}
T f \in V, \quad a(T f, v)=r(f, v), & \forall v \in V, \\
T_{h} f \in V_{h}, \quad a\left(T_{h} f, v_{h}\right)=r\left(f, v_{h}\right), & \forall v_{h} \in V_{h}, \\
T_{*} f \in V, \quad a\left(v, T_{*} f\right)=r(v, f), & \forall v \in V, \\
T_{* h} f \in V_{h}, \quad a\left(v_{h}, T_{* h} f\right)=r\left(v_{h}, f\right), & \forall v_{h} \in V_{h} .
\end{array}
$$

We further assume that

$$
\lim _{h \rightarrow 0}\left|T-T_{h}\right|=0
$$

where $|\cdot|$ denotes the operator norm on $H$. This hypothesis implies $T$ is compact since $T_{h}$ is compact. Note that $a(T u, v)=a\left(u, T_{*} v\right)$ for all $u, v \in V$.

As in Section 3, it is easily seen that the eigenvalues of (4.1) are the reciprocals of the eigenvalues of $T$ and that the eigenvectors of (4.1) are the same as the eigenvectors of $T$.

Let $\mu$ be a nonzero eigenvalue of $T$ with algebraic multiplicity $m$ and let $E$ be the space of generalized eigenvectors of $T$ corresponding to $\mu . \bar{\mu}$ will be an eigenvalue of $T_{*}$ with algebraic multiplicity $m$. To see this, we first note that

$$
T_{* / V}=I\left(T_{/ V}\right) I^{-1}
$$

where $I: V^{*} \rightarrow V$ is defined by $a(v, I \phi)=\overline{\phi(v)}, \forall v \in V, \phi \in V^{*}$ and $(T / V)^{*}$ : $V^{*} \rightarrow V^{*}$ is the usual $V$-adjoint of $T_{/ V}$ considered as a continuous linear operator on $V$. From standard results on adjoints we see that $\bar{\mu}$ is an eigenvalue of $\left(T_{/ V}\right)^{*}$ with algebraic multiplicity $m$. The same result for $T_{*}$ now follows from (4.9). Let $E_{*}$ be the space of generalized eigenvectors of $T_{*}$ corresponding to $\bar{\mu}$. As a consequence of (4.8), we know that exactly $m$ eigenvalues of $T_{h}$ converge to $\mu$. Denote these by $\mu_{1 h}, \mu_{2 h}, \ldots, \mu_{m h}$. We are now ready to state the main theorem of this section. 
THEOREM 4.1. There are two constants $C$ and $h_{0}>0$ such that, for $h<h_{0}$,

$$
\begin{gathered}
\left|\mu-\frac{1}{m} \sum_{i=1}^{m} \mu_{i h}\right| \leqslant C\left\{\sup _{\substack{u \in E \\
|u|=1}} \sup _{\substack{v \in E_{*} \\
|v|=1}}\left|r\left(\left(T-T_{h}\right) u, v\right)\right|\right. \\
\left.\quad+\left|\left(T-T_{h}\right)_{/ E}\right| \cdot\left|\left(T_{*}-T_{* h}\right)_{/ E_{*}}\right|\right\} \\
\equiv C \delta_{h}, \\
\quad\left|\mu^{-1}-\frac{1}{m} \sum_{i=1}^{m} \mu_{i h}^{-1}\right|<C \delta_{h}, \\
\left|\mu-\mu_{j h}\right|^{\alpha} \leqslant C \delta_{h}, \quad j=1,2, \ldots, m,
\end{gathered}
$$

where, for example, $\left|\left(T-T_{h}\right)_{/ E}\right|=\sup _{u \in E ;|u|=1}\left|\left(T-T_{h}\right) u\right|$.

Proof. Let $\Gamma$ be a circle centered at $\mu$ which lies in the resolvent set of $T$ and which encloses no other points in the spectrum of $T$, and let

$$
\begin{aligned}
P=\frac{1}{2 \pi i} \int_{\Gamma}(z-T)^{-1} d z, & P_{h}=\frac{1}{2 \pi i} \int_{\Gamma}\left(z-T_{h}\right)^{-1} d z, \\
P_{*}=\frac{1}{2 \pi i} \int_{\bar{\Gamma}}\left(z-T_{*}\right)^{-1} d z, & P_{* h}=\frac{1}{2 \pi i} \int_{\bar{\Gamma}}\left(z-T_{* h}\right)^{-1} d z
\end{aligned}
$$

be the spectral projections associated with $T$ and $\mu, T_{h}$ and $\mu_{1 h}, \mu_{2 h}, \ldots, \mu_{m h}, T_{*}$ and $\bar{\mu}$, and $T_{* h}$ and $\bar{\mu}_{1 h}, \bar{\mu}_{2 h}, \ldots, \bar{\mu}_{m h}$, respectively; here $\bar{\Gamma}$ is the conjugate cir of $\bar{\Gamma}$ (positively oriented). From (4.8) we see that $\Lambda_{h}=P_{h / E}: E \rightarrow E_{h}$ is a bijection for small $h$ and that $\Lambda_{h}^{-1}$ is uniformly bounded in $h$ :

$$
\left|\Lambda_{h}^{-1}\right|<C, \quad \forall h<h_{0} .
$$

Now we define $\hat{T}=T_{/ E}: E \rightarrow E$ and $\hat{T}_{h}=\Lambda_{h}^{-1} T_{h} \Lambda_{h}: E \rightarrow E$. The spectrum of $\hat{T}$ is $\{\mu\}$ and that of $\hat{T}_{h}$ is $\left\{\mu_{1 h}, \mu_{2 h}, \ldots, \mu_{m h}\right\}$. It is easily seen that $P(H)=E$, $P_{*}(H)=E_{*}, P_{h}(H)=E_{h} \equiv$ the direct sum of the generalized eigenspaces of $T_{h}$ corresponding to $\mu_{1 h}, \mu_{2 h}, \ldots, \mu_{m h}$, and $P_{* h}(H)=E_{* h} \equiv$ the direct sum of the generalized eigenspaces of $T_{* h}$ corresponding to $\bar{\mu}_{1 h}, \bar{\mu}_{2 h}, \ldots, \bar{\mu}_{m h}$. Finally, we note that $a(P u, v)=a\left(u, P_{*} v\right), \forall u, v \in V$, and $r\left(P_{h} u, v\right)=r\left(u, P_{* h} v\right), \forall u, v \in H$.

By standard estimates (see, e.g., [45, pp. 80-81]), we have

$$
\begin{gathered}
\left|\mu-\frac{1}{m} \sum_{i=1}^{m} \mu_{i h}\right|<C\left|\hat{T}-\hat{T}_{h}\right|, \\
\left|\mu^{-1}-\frac{1}{m} \sum_{i=1}^{m} \mu_{i h}^{-1}\right|<C\left|\hat{T}-\hat{T}_{h}\right|, \\
\left|\mu-\mu_{j h}\right|^{\alpha}<C\left|\hat{T}-\hat{T}_{h}\right|, \quad j=1,2, \ldots, m,
\end{gathered}
$$

where $|\cdot|$ is the operator norm on $E$ (corresponding to the vector norm $|\cdot|$ on $H$ ).

Using (4.3), the fact that $E$ is finite-dimensional, and the properties of $P$, we see 
that

$$
\begin{aligned}
& \left|\hat{T}-\hat{T}_{h}\right|=\sup _{\substack{u \in E \\
|u|=1}}\left|\left(\hat{T}-\hat{T}_{h}\right) u\right|<C \sup _{\substack{u \in E \\
\|u\|=1}}\left\|\left(\hat{T}-\hat{T}_{h}\right) u\right\| \\
& <C \sup _{\substack{u \in E \\
\|u\|=1}} \sup _{\substack{v \in V \\
\|v\|=1}}\left|a\left(\left(\hat{T}-\hat{T}_{h}\right) u, v\right)\right|=C \sup _{\substack{u \in E \\
\|u\|=1}} \sup _{\substack{v \in V \\
\|v\|=1}}\left|a\left(\left(\hat{T}-\hat{T}_{h}\right) u, P_{*} v\right)\right| \\
& <C \sup _{\substack{u \in E \\
\|u\|=1}} \sup _{\substack{v \in E_{i} \\
\|v\|=1}}\left|a\left(\left(\hat{T}-\hat{T}_{h}\right) u, v\right)\right|<C \sup _{\substack{u \in E \\
|u|=1}} \sup _{\substack{v \in E_{*} \\
|v|=1}}\left|a\left(\left(\hat{T}-\hat{T}_{h}\right) u, T_{*} v\right)\right| .
\end{aligned}
$$

It follows from the definition of $T_{*}$ and the properties of $P_{h}$ and $\Lambda_{h}$ that

$$
\begin{aligned}
& a\left(\left(\hat{T}-\hat{T}_{h}\right) u, T_{*} v\right)=r\left(\left(\hat{T}-\hat{T}_{h}\right) u, v\right) \\
& \quad=r\left(\left(T-T_{h}\right) u, v\right)+r\left(\left(\Lambda_{h}^{-1} P_{h}-I\right)\left(T-T_{h}\right) u, v\right) \\
& \quad=r\left(\left(T-T_{h}\right) u, v\right)+r\left(\left(\Lambda_{h}^{-1} P_{h}-I\right)\left(T-T_{h}\right) u, v-P_{* h} v\right) .
\end{aligned}
$$

Theorem 4.1 now follows from (4.14), (4.15), (4.16), (4.13), and $\left|\left(P_{*}-P_{* h}\right) / E\right|<$ $C\left|\left(T_{*}-T_{* h}\right) / E\right|$.

Let us turn now briefly to eigenvector estimates.

THEOREM 4.2. There is a constant $C$ such that

$$
\hat{\delta}\left(E, E_{h}\right)<C\left|\left(T-T_{h}\right)_{/ E}\right|
$$

where $\hat{\delta}\left(E, E_{h}\right)$ is the gap with respect to the $H$-norm and $|\cdot|$ is the operator norm corresponding to the $H$-norm.

Proof. A minor modification of the techniques in [36] yields this result.

5. Problems of Type (Q1). In this section we consider problems of type (Q1) that do not satisfy the full Brezzi hypotheses ((3.12)-(3.16), (3.4)). We will, however, make other alternate hypotheses. Throughout the section we suppose $H$ is a Hilbert space with $X \subset H$ continuously and suppose that $r(\cdot, \cdot)$ is a bounded sesquilinear form on $H \times H$. We also suppose that $b(v, q)=0, \forall v \in X$, implies $q=0$ and that $b\left(v_{h}, q_{h}\right)=0, \forall v_{h} \in X_{h}$, implies $q_{h}=0$.

We consider now the associated source problem and approximate source problem as well as their adjoints. These are defined as follows:

For $g \in H$,

$$
\begin{aligned}
& A g \in X, B g \in W, \\
& \begin{cases}a(A g, v)+\overline{b(v, B g)}=r(g, v), & \forall v \in X, \\
b(A g, q)=0, & \forall q \in W ;\end{cases} \\
& A_{h} g \in X_{h}, B_{h} g \in W_{h}, \\
& \begin{cases}a\left(A_{h} g, v_{h}\right)+\overline{b\left(v_{h}, B_{h} g\right)}=r\left(g, v_{h}\right), & \forall v_{h} \in X_{h}, \\
b\left(A_{h} g, q_{h}\right)=0, & \forall q_{h} \in W_{h} ;\end{cases} \\
& A_{*} g \in X, B_{*} q \in W, \\
& \begin{cases}a\left(v, A_{*} g\right)+b\left(v, B_{*} g\right)=r(v, g), & \forall v \in X, \\
b\left(A_{*} g, q\right)=0, & \forall q \in W ;\end{cases}
\end{aligned}
$$




$$
\begin{aligned}
& A_{* h} g \in X_{h}, B_{* h} q \in W_{h}, \\
& \begin{cases}a\left(v_{h}, A_{* h} g\right)+b\left(v_{h}, B_{* h} g\right)=r\left(v_{h}, g\right), & \forall v_{h} \in X_{h}, \\
b\left(A_{* h} g, q_{h}\right)=0, & \forall q_{h} \in W_{h} .\end{cases}
\end{aligned}
$$

We shall suppose all these problems are uniquely solvable and that the component in $X$ of the solution depends continuously on $g$ (in connection with (5.1), for example, we would assume $\|A g\|_{X} \leqslant C\|g\|_{H}, \forall g \in H$ ). In many practical cases (see Subsection $7 \mathrm{~d}$ ), operators $B, B_{*}$ are uniquely defined but source problems (5.1), (5.3) are not well posed in general.

Let

$$
V=\{v \in X: b(v, q)=0, \forall q \in W\}
$$

and

$$
V_{h}=\left\{v_{h} \in X_{h}: b\left(v_{h}, q_{h}\right)=0, \forall q_{h} \in W_{h}\right\}
$$

and assume

$$
\begin{array}{ll}
\operatorname{Re} a(u, u) \geqslant \alpha\|u\|_{X}^{2}, & \forall u \in V, \\
\operatorname{Re} a\left(u_{h}, u_{h}\right)>0, & \forall u_{h} \in V_{h}, u_{h} \neq 0 \text { and } \forall h .
\end{array}
$$

If $\lambda,(u, p)$ is an eigenpair of $(\mathrm{Q} 1)$, then

$$
\left\{\begin{array}{l}
u \in V, u \neq 0 \\
a(u, v)=\lambda r(u, v), \quad \forall v \in V .
\end{array}\right.
$$

Conversely, if $u$ satisfies (5.5), then there exists a unique $p \in W,(p=\lambda B u)$, such that $\lambda,(u, p)$ is an eigenpair of $(\mathrm{Q} 1)$.

Thus, the eigenvalues of (Q1) can be characterized by a problem of type (5.5). $p$ is the Lagrange multiplier for the constraint $u \in V$ and (5.5) is a constrained version of (Q1).

In a similar way, we see that the eigenvalues of $(\mathrm{Q} 1)_{h}$ can be characterized by the problem

$$
\left\{\begin{array}{l}
\text { Seek } \lambda_{h} \in C, 0 \neq u_{h} \in V_{h} \text { satisfying } \\
a\left(u_{h}, v_{h}\right)=\lambda_{h} r\left(u_{h}, v_{h}\right), \quad \forall v_{h} \in V_{h} .
\end{array}\right.
$$

Problems (5.5) and (5.6) are examples of problems (4.1) and (4.2). With $A, A_{h}$, $A_{*}$, and $A_{* h}$ defined as in (5.1)-(5.4) and $T, T_{h}, T_{*}$, and $T_{* h}$ defined as in (4.4)-(4.7), we immediately see that $T=A, T_{h}=A_{h}, T_{*}=A_{*}$, and $T_{* h}=A_{* h}$. Assume now that

$$
\lim _{h \rightarrow 0}\left\|A-A_{h}\right\|_{H H}=0
$$

where, for an operator $D: Y \rightarrow Z$, we set

$$
\|D\|_{Y Z}=\sup _{\substack{w \in Y \\ w \neq 0}} \frac{\|D w\|_{Z}}{\|w\|_{Y}} .
$$

(4.8) holds and thus all the hypotheses of Theorems 4.1 and 4.2 are satisfied, and we can apply them in the present context. Let $\mu$ be an eigenvalue of $A$ with algebraic multiplicity $m$, let $\mu_{1 h}, \mu_{2 h}, \ldots, \mu_{m h}$ be the eigenvalues of $A_{h}$ converging 
to $\mu$, and set $\lambda=\mu^{-1}, \lambda_{j h}=\mu_{j h}^{-1}, j=1, \ldots, m$. Let $E, E_{*}, E_{h}$, and $E_{* h}$ be the spaces of generalized eigenvectors as defined in Section 4.

THEOREM 5.1. Under hypothesis (5.7) there are constants $C$ and $h_{0}>0$ such that, for $h \leqslant h_{0}$,

$$
\begin{aligned}
& \left|\lambda-\frac{1}{m} \sum_{i=1}^{m} \lambda_{i h}\right| \leqslant C\left\{\left\|\left(A-A_{h}\right)_{/ E}\right\|_{H X}\left\|\left(A_{*}-A_{* h}\right)_{/ E_{*}}\right\|_{H X}\right.
\end{aligned}
$$

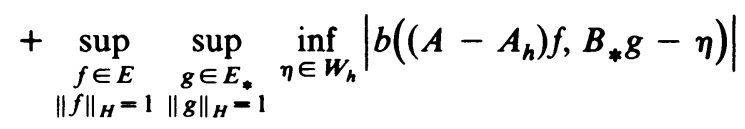

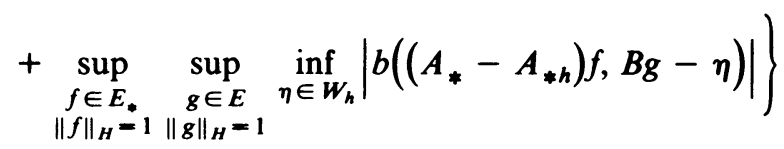

$$
\begin{aligned}
& \leqslant C\left\{\left\|\left(A-A_{h}\right)_{/ E}\right\|_{H X}\left\|\left(A_{*}-A_{* h}\right)_{/ E_{*}}\right\|_{H X}\right. \\
& +\left\|\left(A-A_{h}\right) / E\right\|_{H X} \sup _{\substack{g \in E_{*} \\
\|g\|_{H}=1}} \inf _{\eta \in W_{h}}\left\|B_{*} g-\eta\right\|_{W} \\
& \left.+\left\|\left(A_{*}-A_{* h}\right) \sum_{E_{*}}\right\|_{H X} \sup _{\substack{g \in E \\
\boldsymbol{g} \|_{H}=1}} \inf _{\eta \in W_{h}}\|B g-\eta\|_{W}\right\} \\
& \leqslant C\left\{\left\|\left(A-A_{h}\right)_{/ E}\right\|_{H X}\left\|\left(A_{*}-A_{* h}\right)_{/ E_{*}}\right\|_{H X}\right. \\
& +\left\|\left(A-A_{h}\right)_{/ E}\right\|_{H X}\left\|\left(B_{*}-B_{* h}\right)_{/ E_{*}}\right\|_{H W} \\
& \left.+\left\|\left(A_{*}-A_{* h}\right)_{/ E_{*}}\right\|_{H X}\left\|\left(B-B_{h}\right)_{/ E}\right\|_{H W}\right\} .
\end{aligned}
$$

Proof. We apply Theorem 4.1 with $T=A, T_{*}=A_{*}, T_{h}=A_{h}$, and $T_{* h}=A_{* h}$. It is immediate that the second term on the right side of $(4.10)$ is bounded by the right side of (5.8). It remains to consider

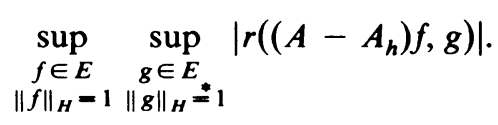

Let $f, g \in H$. Adding the two equations in (5.3), we have

$$
r(v, g)=a\left(v, A_{*} g\right)+b\left(v, B_{*} g\right)+\overline{b\left(A_{*} g, q\right)}, \quad \forall(v, q) \in X \times W
$$

Setting $v=\left(A-A_{h}\right) f$ and $q=\left(B-B_{h}\right) f$, we obtain

$$
\begin{aligned}
r\left(\left(A-A_{h}\right) f, g\right)= & a\left(\left(A-A_{h}\right) f, A_{*} g\right)+b\left(\left(A-A_{h}\right) f, B_{*} g\right) \\
& +\overline{b\left(A_{*} g,\left(B-B_{h}\right) f\right)} .
\end{aligned}
$$


Next, we note that subtraction of Eqs. (5.2) from (5.1) (with $g$ replaced by $f$ ) yields

$$
\begin{aligned}
a\left(\left(A-A_{h}\right) f, v_{h}\right)+\overline{b\left(v_{h},\left(B-B_{h}\right) f\right)}+b\left(\left(A-A_{h}\right) f, q_{h}\right) & =0, \\
\forall\left(v_{h}, q_{h}\right) & \in X_{h} \times W_{h} .
\end{aligned}
$$

Now, combining (5.9) and (5.10), we have

$$
\begin{aligned}
r\left(\left(A-A_{h}\right) f, g\right)=a & \left(\left(A-A_{h}\right) f, A_{*} g-v_{h}\right)+b\left(\left(A-A_{h}\right) f, B_{*} g-q_{h}\right) \\
& +\overline{b\left(A_{*} g-v_{h},\left(B-B_{h}\right) f\right)}, \quad \forall\left(v_{h}, q_{h}\right) \in X_{h} \times W_{h} .
\end{aligned}
$$

Setting $v_{h}=A_{* h} g$ in (5.11) and using (5.3) and (5.4), we find

$$
\begin{aligned}
r\left(\left(A-A_{h}\right) f, g\right)=a & \left(\left(A-A_{h}\right) f,\left(A_{*}-A_{* h}\right) g\right)+b\left(\left(A-A_{h}\right) f, B_{*} g-q_{h}\right) \\
& +b\left(\left(A_{*}-A_{* h}\right) g, B f-\tilde{q}_{h}\right), \quad \forall q_{h}, \tilde{q}_{h} \in W_{h} .
\end{aligned}
$$

(5.8) now follows immediately from Theorem 4.1 and (5.12).

THEOREM 5.2. Under hypothesis (5.7) there is a constant $C$ such that

$$
\hat{\delta}\left(E, E_{h}\right) \leqslant C\left\|\left(A-A_{h}\right)_{/ E}\right\|_{H H},
$$

where $\hat{\delta}\left(E, E_{h}\right)$ is the $H$-gap between $E$ and $E_{h}$.

Proof. This result is a direct consequence of Theorem 4.2.

Remark. It is easily seen that Theorems 5.1 and 5.2 are valid in the more general context in which the spaces $X$ and $W$ are allowed to depend on $h(X=X(h)$ and $W=W(h)$ ), but $V$ is independent of $h$, and the forms $a$ and $b$ are bounded for each $h$ but are not required to be bounded uniformly in $h$. This remark is used in Subsection 7c.

6. Problems of Type (Q2). In this section we consider problems of type (Q2) that do not satisfy the full Brezzi hypotheses ((3.12)-(3.16), (3.4)). As in Section 5 we make alternate hypotheses.

We assume $H$ and $G$ are complex Hilbert spaces with $X \subset H$ continuously and $W \subset G$ compactly. We then suppose that $s(p, q)=(p, q)_{G}$ and that $a(\cdot, \cdot)$ is a bounded sesquilinear form on $H \times H$ satisfying $\operatorname{Re} a(u, u)>0 \forall 0 \neq u \in H$.

Consider now the associated source problem and approximate source problem and their adjoints. These are defined as follows: For $g \in G$,

$$
\begin{aligned}
& A g \in X, B g \in W \\
& \begin{cases}a(A g, v)+\overline{b(v, B g)}=0, & \forall v \in X, \\
b(A g, q)=-(g, q)_{G}, & \forall q \in W ;\end{cases} \\
& A_{h} g \in X_{h}, B_{h} g \in W_{h} \\
& \begin{cases}a\left(A_{h} g, v_{h}\right)+\overline{b\left(v_{h}, B_{h} g\right)}=0, & \forall v_{h} \in X_{h}, \\
b\left(A_{h} g, q_{h}\right)=-\left(g, q_{h}\right)_{G},\end{cases} \\
& A_{*} g \in X, B_{*} g \in W \\
& \begin{cases}a\left(v, A_{*} g\right)+b\left(v, B_{*} g\right)=0, & \forall v \in X, \\
\frac{b\left(A_{*} g, q\right)}{2}=-(q, g)_{G}, & \forall q \in W,\end{cases}
\end{aligned}
$$




$$
\begin{aligned}
& A_{* h} g \in X_{h}, B_{* h} g \in W_{h} \\
& \begin{cases}a\left(v_{h}, A_{* h} g\right)+b\left(v_{h}, B_{* h} g\right)=0, & \forall v_{h} \in X_{h}, \\
\overline{b\left(A_{* h} g, q_{h}\right)}=-\left(q_{h}, g\right)_{G}, & \forall q_{h} \in W_{h} .\end{cases}
\end{aligned}
$$

We suppose all these problems are uniquely solvable.

In addition we assume

$$
\lim _{h \rightarrow 0}\left\|B-B_{h}\right\|_{G G}=0 .
$$

This relation implies that $B: G \rightarrow G$ is compact. We note that $B_{*}=B^{*}$, the usual $G$-adjoint of $B$.

The eigenvalues of $(\mathrm{Q} 2)$ can be characterized in terms of the operator $B$. In fact, if $\lambda,(u, p)$ is an eigenpair of (Q2), then $\lambda B p=p, p \neq 0$, and if $\lambda B p=p, p \neq 0$, then there is a $u \in X$ such that $\lambda,(u, p)$ is an eigenpair of (Q2). Thus, the eigenvalues of $(\mathrm{Q} 2)$ are the reciprocals of the eigenvalues of $B$. In a similar way, we see that the eigenvalues of $(\mathrm{Q} 2)_{h}$ are the reciprocals of the eigenvalues of $B_{h}$.

We now apply Theorem 2.1 to the operator $B$ and the family of operators $\left\{B_{h}\right\}$ on the space $G$. Suppose $\lambda^{-1}$ has algebraic multiplicity $m$ and let

$$
E=\operatorname{Ker}\left(\left(\lambda^{-1}-B\right)^{\alpha}\right) \text { and } E_{*}=\operatorname{Ker}\left(\left(\bar{\lambda}^{-1}-B^{*}\right)^{\alpha}\right) \text {, }
$$

where $\alpha$ is the ascent of $\lambda^{-1}-B$. Let $\lambda_{1 h}^{-1}, \lambda_{2 h}^{-1}, \ldots, \lambda_{m h}^{-1}$ be the $m$ eigenvalues of $B_{h}$ that converge to $\lambda^{-1}$ and let $\hat{\lambda}_{h}=(1 / m) \sum_{i=1}^{m} \lambda_{i h}$.

THEOREM 6.1. Under hypothesis (6.5) there are two constants $C$ and $h_{0}>0$ such that, for $h \leqslant h_{0}$,

$$
\begin{aligned}
& \left|\lambda-\hat{\lambda}_{h}\right| \leqslant C\left\{\left\|\left(A-A_{h}\right)_{/ E}\right\|_{G H}\left\|\left(A_{*}-A_{* h}\right)_{/ E_{*}}\right\|_{G H}\right.
\end{aligned}
$$

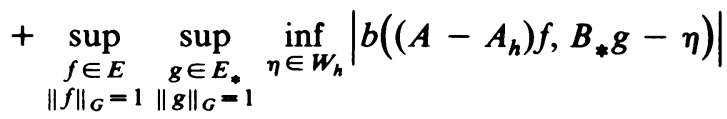

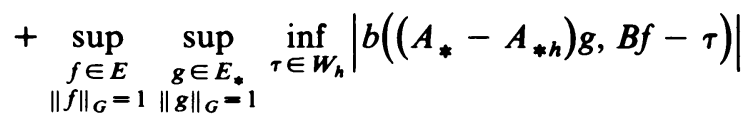

$$
\begin{aligned}
& \left.+\left\|\left(B-B_{h}\right)_{/ E}\right\|_{G G}\left\|\left(B_{*}-B_{* h}\right)_{/ E_{*}}\right\|_{G G}\right\} \\
& \leqslant C\left\{\left\|\left(A-A_{h}\right)_{/ E}\right\|_{G H}\left\|\left(A_{*}-A_{* h}\right)_{/ E_{*}}\right\|_{G H}\right. \\
& +\left\|\left(A-A_{h}\right)_{/ E}\right\|_{G X}\left\|\left(B_{*}-B_{* h}\right)_{/ E *}\right\|_{G W} \\
& +\left\|\left(A_{*}-A_{* h}\right)_{/ E_{*}}\right\|_{G X}\left\|\left(B^{*}-B_{h}\right)_{/ E}\right\|_{G W} \\
& \left.+\left\|\left(B-B_{h}\right)_{/ E}\right\|_{G G}\left\|\left(B_{*}-B_{* h}\right)_{/ E_{*}}\right\|_{G G}\right\} \text {. }
\end{aligned}
$$


Proof. For $f, g \in G$ we consider $\left(\left(B-B_{h}\right) f, g\right)_{G}$. From (6.3) we have

$$
(q, g)_{G}=-a\left(v, A_{*} g\right)-b\left(v, B_{*} g\right)-\overline{b\left(A_{*} g, q\right)}, \quad \forall(v, q) \in X \times W .
$$

Setting $q=\left(B-B_{h}\right) f$ and $v=\left(A-A_{h}\right) f$, we obtain

$$
\begin{aligned}
\left(\left(B-B_{h}\right) f, g\right)_{G}= & -a\left(\left(A-A_{h}\right) f, A_{*} g\right)-b\left(\left(A-A_{h}\right) f, B_{*} g\right) \\
& -\overline{b\left(A_{*} g,\left(B-B_{h}\right) f\right)} .
\end{aligned}
$$

Subtraction of (6.2) from (6.1) (with $g$ replaced by $f$ ) yields

$$
\begin{aligned}
& a\left(\left(A-A_{h}\right) f, v_{h}\right)+\overline{b\left(v_{h},\left(B-B_{h}\right) f\right)}+b\left(\left(A-A_{h}\right) f, q_{h}\right)=0, \\
& \forall\left(v_{h}, q_{h}\right) \in X_{h} \times W_{h} .
\end{aligned}
$$

Now, combining (6.7) and (6.8), we have

$$
\begin{aligned}
\left(\left(B-B_{h}\right) f, g\right)_{G}= & -a\left(\left(A-A_{h}\right) f, A_{*} g-v_{h}\right)-b\left(\left(A-A_{h}\right) f, B_{*} g-q_{h}\right) \\
& -\overline{b\left(A_{*} g-v_{h},\left(B-B_{h}\right) f\right)}, \quad \forall\left(v_{h}, q_{h}\right) \in W_{h} \times W_{h} .
\end{aligned}
$$

Setting $v_{h}=A_{* h} g$ in (6.9) and using (6.3) and (6.4), we get

$$
\begin{aligned}
\left(\left(B-B_{h}\right) f, g\right)_{G}= & -a\left(\left(A-A_{h}\right) f,\left(A_{*}-A_{* h}\right) g\right) \\
& -b\left(\left(A-A_{h}\right) f, B_{*} g-q_{h}\right) \\
& -\overline{b\left(\left(A_{*}-A_{* h}\right) g, B f-\tilde{q}_{h}\right)}, \quad \forall q_{h}, \tilde{q}_{h} \in W_{h} .
\end{aligned}
$$

(6.6) now follows immediately from Theorem 2.1 and (6.10).

THEOREM 6.2. There is a constant $C$ such that

$$
\hat{\delta}\left(E, E_{h}\right) \leqslant C\left\|\left(B-B_{h}\right)_{/ E}\right\|_{G G},
$$

where $\delta\left(E, E_{h}\right)$ is the G-gap between $E$ and $E_{h}$.

Proof. This result follows Theorem 4.2.

Theorem 6.2 provides an error estimate for the error in the approximation of the second component of the eigenfunction $(u, p)$, i.e., an estimate for $\left\|p-p_{h}\right\|_{G}$. We now present a result giving error estimates for both components.

Introduce the sesquilinear forms on $(X \times W) \times(X \times W)$ defined by

$$
A(u, p ; v, q)=a(u, v)+\overline{b(v, p)}+b(u, q) \text { and } B(u, p ; v, q)=-(p, q)_{G}
$$

(cf. Section 3) and let $\tau, \tau_{h}: X \times W \rightarrow X \times W$ be the operators defined by

$$
\begin{aligned}
& \left\{\begin{array}{l}
\tau(u, p) \in X \times W, \\
A(\tau(u, p) ; v, q)=B(u, p ; v, q), \forall(v, q) \in X \times W,
\end{array}\right. \\
& \left\{\begin{array}{l}
\tau_{h}(u, p) \in X_{h} \times W_{h}, \\
A\left(\tau_{h}(u, p) ; v, q\right)=B(u, p ; v, q), \quad \forall(v, q) \in X_{h} \times W_{h} .
\end{array}\right.
\end{aligned}
$$

It is easily seen that the eigenvalues of $\left(Q_{2}\right)$ are the reciprocals of the eigenvalues of $\tau$ and that $\tau$ and $\left(Q_{2}\right)$ have the same eigenfunctions. The relation between the eigenvalues and eigenvectors of $(\mathrm{Q} 2)_{h}$ and $\tau_{h}$ is the same. We now assume

$$
\lim _{h \rightarrow 0}\left\|\tau-\tau_{h}\right\|_{X \times W, X \times W}=0
$$


We note that $\tau(u, p)$ does not depend on $u$ and also that

$$
\tau(u, p)=(A p, B p), \quad \forall p \in W .
$$

Suppose $\lambda^{-1}$ is an eigenvalue of $\tau$ with algebraic multiplicity $m$ and let $E=$ $\operatorname{Ker}\left(\left(\lambda^{-1}-\tau\right)^{\alpha}\right)$ where $\alpha$ is the ascent of $\lambda^{-1}-\tau$. Let $\lambda_{1 h}^{-1}, \lambda_{2 h}^{-1}, \ldots, \lambda_{m h}^{-1}$ be the eigenvalues of $\tau_{h}$ converging to $\lambda^{-1}$ and let $E_{h}$ be the direct sum of the generalized eigenspaces of $\tau_{h}$ corresponding to $\lambda_{1 h}^{-1}, \lambda_{2 h}^{-1}, \ldots, \lambda_{m h}^{-1}$.

THEOREM 6.3. Under hypothesis (6.13) there is a constant $C$ such that

$$
\hat{\delta}\left(E, E_{h}\right) \leqslant C\left\|\left(\tau-\tau_{h}\right)_{/ E}\right\|_{X \times W, X \times W},
$$

where $\hat{\delta}\left(E, E_{h}\right)$ is the $X \times W$-gap between $E$ and $E_{h}$.

Proof. This result is a direct consequence of Theorem 2.2.

There is a subclass of problems of type (Q2) for which it is possible to improve the above results. Suppose

$$
V_{h} \subset V,
$$

where

$$
V_{h}=\left\{v \in X_{h}: b(v, \phi)=0, \forall \phi \in W_{h}\right\}
$$

and

$$
V=\{v \in X: b(v, \phi)=0, \forall \phi \in W\}
$$

and that there exists an operator $\Pi_{h}: Y=\operatorname{span}\{A g\}_{g \in G} \rightarrow X_{h}$ satisfying

$$
b\left(y-\Pi_{h} y, \phi\right)=0, \quad \forall y \in Y \text { and } \forall \phi \in W_{h} .
$$

The existence of a family $\left\{\Pi_{h}\right\}$ satisfying (6.15) and which is in addition uniformly bounded with respect to the $X$-norm, is closely related to the condition that there is a $k_{0}>0$, independent of $h$, such that

$$
\sup _{v \in X_{h}} \frac{|b(v, \phi)|}{\|v\|_{X}} \geqslant k_{0}\|\phi\|_{W}, \quad \forall \phi \in W_{h},
$$

(see [6], [15], [17]). $\Pi_{h}$ has been constructed for several mixed methods (CiarletRaviart [10], Herrmann-Miyoshi [20], [21], [29], and Herrmann-Johnson [20], [21], [25], for example). Condition (6.14) is relatively special. It holds, for example, in the Herrmann-Johnson method; cf. Subsection $7 b$ (ii).

We assume here, for the sake of simplicity, that the form $a$ is hermitian. Then $A=A_{*}$ and $B=B_{*}$. It follows from (6.1), (6.2), (6.14), and (6.15) that

$$
b\left(A_{h} g-\Pi_{h} A g, \phi\right)=0, \quad \forall g \in G \text { and } \forall \phi \in W \text {. }
$$

Now, using (6.10) and (6.16), we obtain

$$
\begin{aligned}
\left(\left(B-B_{h}\right) f, g\right)_{G}= & -a\left(\left(A-A_{h}\right) f,\left(A-A_{h}\right) g\right)-b\left(A f-\Pi_{h} A f, B g-\eta\right) \\
& -b\left(A g-\Pi_{h} A g, B f-\tau\right), \quad \forall \eta, \tau \in W_{h} .
\end{aligned}
$$

Combining (6.17) and Theorem 2.1, we have in this case 
THEOREM 6.4. Under the assumptions above, there exists a constant $C$ such that

$$
\begin{aligned}
\left|\lambda-\hat{\lambda}_{h}\right| \leqslant C\left\{\left\|\left(A-A_{h}\right) / E\right\|_{G H}^{2}\right. & \\
+ & \sup _{\substack{f \in E \\
\|f\|_{G}=1}} \sup _{\substack{g \in E \\
\|g\|_{G}=q}} \inf _{\eta \in W_{h}}\left|b\left(A f-\Pi_{h} A f, B g-\eta\right)\right| \\
& \left.+\left\|\left(B-B_{h}\right)_{/ E}\right\|_{G G}^{2}\right\} .
\end{aligned}
$$

Remark. It is easily seen that Theorem 6.4 holds in the more general context in which $W$ is a Banach space, the space $X$ is allowed to depend on $h(X=X(h))$, and the form $b$ is bounded on $X(h) \times W$ for each $h$ but is not required to be bounded uniformly in $h$. This remark is used in Subsection 7b(ii).

7. Applications. In this section we apply the results of Sections 3-6 to a variety of methods of mixed and hybrid type.

a. In this subsection we discuss a method which fits within the framework of Section 3. Consider the eigenvalue problem

$$
\begin{cases}-\Delta \psi=\lambda \psi & \text { in } \Omega \\ \psi=0 & \text { on } \Gamma=\partial \Omega\end{cases}
$$

where $\Omega$ is a convex polygon in $\mathbf{R}^{2}$. We then consider the following mixed formulation of (7.1):

Seek $\lambda,(u, \psi) \in H(\operatorname{div}, \Omega) \times L^{2}(\Omega)$ satisfying

$$
\begin{cases}\int_{\Omega} u \bar{v} d x+\int_{\Omega} \psi \operatorname{div} \bar{v} d x=0, & \forall v \in H(\operatorname{div}, \Omega), \\ \int_{\Omega} \phi \operatorname{div} \bar{u} d x=-\lambda \int_{\Omega} \psi \bar{\phi} d x, & \forall \phi \in L^{2}(\Omega) .\end{cases}
$$

If $\lambda, \psi$ is an eigenpair of (7.1) and $u=\operatorname{grad} \psi$, then $\lambda,(u, \psi)$ is an eigenpair of (7.2), and if $\lambda,(u, \psi)$ is an eigenpair of (7.2), then $\lambda, \psi$ is an eigenpair of (7.1) and $u=\operatorname{grad} \psi$.

The eigenvalue problem (7.2) is of type (Q2) with $X=H(\operatorname{div}, \Omega), W=L^{2}(\Omega)$, $a(u, v)=\int_{\Omega} u \bar{v} d x, b(u, \psi)=\int_{\Omega} \operatorname{div} u \bar{\psi} d x$, and $s(\psi, \phi)=\int_{\Omega} \psi \bar{\phi} d x$. If we set $V=$ $\{v \in X: b(v, q)=0, \forall q \in W\}=\{v \in H(\operatorname{div}, \Omega): \operatorname{div} v=0\}$, it is immediate that (3.12) and (3.13) are satisfied. (In this subsection we use the norm $\|u\|=\left(\int_{\Omega}|u|^{2} d x\right.$ $\left.+\int_{\Omega}|\operatorname{div} u|^{2} d x\right)^{1 / 2}$ on $H(\operatorname{div}, \Omega)$.) We next describe the finite-dimensional subspaces used in the approximation scheme. Following [40], we begin by introducing the space $\hat{Q}$ associated with the unit right triangle $\hat{T}$ in the $(\xi, \eta)$-plane whose vertices are $\hat{a}_{1}=(1,0), \hat{a}_{2}=(0,1)$, and $\hat{a}_{3}=(0,0)$. For $K>0$ an even integer, define $\hat{Q}$ to be the space of all functions $\hat{q}$ of the form

$$
\left\{\begin{array}{l}
\hat{q}_{1}=\operatorname{pol}_{K}(\xi, \eta)+\alpha_{0} \xi^{K+1}+\alpha_{1} \xi^{K} \eta+\cdots+\alpha_{K / 2} \sigma^{(K / 2)+1} \eta^{K / 2} \\
\hat{q}_{2}=\operatorname{pol}_{K}(\xi, \eta)+\beta_{0} \eta^{K+1}+\beta_{1} \xi^{K}+\cdots+\beta_{K / 2} \xi^{K / 2} \eta^{K / 2+1}
\end{array}\right.
$$

with $\sum_{i=0}^{K / 2}(-1)^{i}\left(\alpha_{i}-\beta_{i}\right)=0$, where $\operatorname{pol}_{K}(\xi, \eta)$ denotes any polynomial of degree $K$ in the two variables $\xi, \eta$. For $K>1$ an odd integer, define $\hat{Q}$ to be the space of all functions $\hat{q}$ of the form

$$
\left\{\begin{array}{l}
\hat{q}_{1}=\operatorname{pol}_{K}(\xi, \eta)+\alpha_{0} \xi^{k+1}+\alpha_{1} \xi^{K} \eta+\cdots+\alpha_{(K+1) / 2} \xi^{(K+1) / 2} \eta^{(K+1) / 2} \\
\hat{q}_{2}=\operatorname{pol}_{K}(\xi, \eta)+\beta_{0} \eta^{K+1}+\beta_{1} \xi \eta^{K}+\cdots+\beta_{(K+1) / 2} \xi^{(K+1) / 2} \eta^{(K+1) / 2}
\end{array}\right.
$$


with

$$
\sum_{i=0}^{(K+1) / 2}(-1)^{i} \alpha_{i}=\sum_{i=0}^{(K+1) / 2}(-1)^{i} \beta_{i}=0 .
$$

Now consider any triangle $T$ in the $\left(x_{1}, x_{2}\right)$-plane whose vertices are denoted by $a_{i}, 1<i \leqslant 3$. Let $F_{T}: \hat{x} \rightarrow F_{T}(\hat{x})=B_{T} \hat{x}+b_{T}, B_{T} \in \mathcal{L}\left(\mathbf{R}^{2}\right), b_{T} \in \mathbf{R}^{2}$ be the unique invertible affine mapping such that $F_{T}\left(\hat{a}_{i}\right)=a_{i}, 1 \leqslant i<3$. With each vector-valued function $\hat{v}=\left(\hat{v}_{1}, \hat{v}_{2}\right)$ defined on $\hat{T}$ we associate the function $v$ defined on $T$ by

$$
v=\frac{1}{J_{T}} B_{T} \hat{v} \circ F_{T}^{-1}
$$

where $J_{T}=\operatorname{det} B_{T}$.

For $0<h \leqslant 1$, let $\tau_{h}$ be a triangulation of $\bar{\Omega}$ made up of triangles $T$ whose diameters are less than or equal to $h$. We assume the family $\left\{\tau_{h}\right\}$ satisfies the minimal angle condition, i.e., there is a constant $\sigma>0$ such that

$$
\max _{T \in \tau_{h}} \frac{h_{T}}{\rho_{T}}<\sigma, \quad \forall h,
$$

where $h_{T}$ is the diameter of $T$ and $\rho_{T}$ is the diameter of the largest circle contained in $T$. We now let

$$
X_{h}=\left\{v \in H(\operatorname{div}, \Omega): \forall T \in \tau_{h}, v /{ }_{T} \in Q_{T}\right\},
$$

where

$$
Q_{T}=\{v \in H(\operatorname{div}, T): \hat{v} \in \hat{Q}\} \quad \text { and } \quad W_{h}=\left\{\phi \in L^{2}(\Omega): \forall T \in \tau_{h}, \phi / T \in P_{k}\right\} \text {, }
$$

where $P_{K}$ is the space of all polynomials of degree $K$ in the variables $x_{1}, x_{2}$.

We now consider the approximation method (Q2) $\left(\right.$ or (3.5)) with $X_{h}$ and $W_{h}$ as above. (3.14)-(3.16) are shown to be satisfied in Theorems 3 and 4 of [40]. We can thus apply the results of Section 3 to this method.

With this method we obtain an approximation to $\lambda, \psi$ and $u=\operatorname{grad} \psi$.

From Theorem 3.1 (or from (3.17)) and Theorem 3 in [40] we have (using the notations from Section 3)

$$
\begin{aligned}
\left|\lambda-\lambda_{i h}\right| & \leqslant C \varepsilon_{h}^{2}=C \sup _{\substack{(u, \psi) \in E \\
\|u\|+\|\psi\|_{0, \Omega}=1}} \inf _{\substack{u_{h} \in X_{h} \\
\psi_{h} \in W_{h}}}\left(\left\|u-u_{h}\right\|+\left\|\psi-\psi_{h}\right\|_{0, \Omega}\right)^{2} \\
& =C h^{2 K+2},
\end{aligned}
$$

provided $\psi \in H^{K+2}(\Omega)$. For the eigenvector error we obtain $\hat{\delta}\left(E, E_{h}\right)<C h^{K+1}$, where $\hat{\delta}\left(E, E_{h}\right)$ is the gap between $E$ and $E_{h}$ in the norm of $H(\operatorname{div}, \Omega) \times L^{2}(\Omega)$.

Remark. We obtain the same estimates when we use the finite-dimensional subspaces described in [18].

b. In this subsection we study mixed methods for the approximation of eigenvalues of 4th order problems. Eigenvalue estimates for these methods were first obtained by Canuto [9].

(i) Consider the model eigenvalue problem

$$
\begin{cases}\Delta^{2} \psi=\lambda \psi & \text { in } \Omega \\ \psi=\frac{\partial \psi}{\partial n}=0 & \text { on } \Gamma=\partial \Omega\end{cases}
$$


where $\Omega$ is a convex polygon in $\mathbf{R}^{2}$. The mixed method we study here is based on the following formulation of (7.6):

Seek $\lambda,(u, \psi) \in H^{1}(\Omega) \times H_{0}^{1}(\Omega)$ satisfying

$$
\begin{cases}\int_{\Omega} u \bar{v} d x-\int_{\Omega} \overline{\nabla v} \nabla \psi d x=0, & \forall v \in H^{1}(\Omega), \\ -\int_{\Omega} \nabla u \overline{\nabla \phi} d x=-\lambda \int_{\Omega} \psi \bar{\phi} d x, & \forall \phi \in H_{0}^{1}(\Omega),\end{cases}
$$

where $\nabla$ is the gradient operator.

It is not difficult to show that if $\lambda, \psi$ is an eigenpair of (7.6) and $u=-\Delta \psi$, then $\lambda$, $(u, \psi)$ is an eigenpair of (7.7) and if $\lambda,(u, \psi)$ is an eigenpair of (7.7), then $\lambda, \psi$ is an eigenpair of (7.6) and $u=-\Delta \psi$.

The eigenvalue problem (7.7) is of type (Q2) with $X=H^{1}(\Omega), W=H_{0}^{1}(\Omega)$, $a(u, v)=\int_{\Omega} u \bar{v} d x, b(u, \psi)=-\int_{\Omega} \nabla u \bar{\nabla} \psi d x$, and $s(\psi, \phi)=\int_{\Omega} \psi \bar{\phi} d x$. Note that assumption (3.12) is not satisfied here.

Next, we discuss the approximate eigenvalue problems. As in Subsection 7a, let $\left\{\tau_{h}\right\}$ be a triangulation of $\bar{\Omega}$ which satisfies the minimal angle condition and is in addition quasiuniform, i.e., there is a constant $\tau>0$ such that

$$
\max _{T} h_{T} / \min _{T} h_{T}<\tau, \quad \forall h .
$$

Then we consider problem $\left(Q_{2}\right)_{h}$ with

$$
X_{h}=\left\{v \in C^{0}(\bar{\Omega}): v /{ }_{T} \in P_{K}, \forall T \in \tau_{h}\right\}
$$

and $W_{h}=X_{h} \cap H_{0}^{1}(\Omega)$.

With this method we obtain approximations to $\lambda, \psi$ and $u=-\Delta \psi$. This method as applied to source problems was studied in Glowinski [19], Mercier [27], and Ciarlet-Raviart [10].

We analyze this method by means of Theorems 6.1, 6.2, and 6.3. Our problem fits into the framework of Section 6 with $H=G=L^{2}(\Omega)$. Clearly $B_{*}=B$, $A_{*}=A$, and $B$ is selfadjoint. The problems (6.1)-(6.4) are uniquely solvable and $B, B_{h}: L^{2}(\Omega) \rightarrow L^{2}(\Omega)$ are easily seen to be compact. Suppose $K>2$. Then, using the results in Subsection 3a in [15], we have:

$$
\begin{gathered}
\left\|\left(A-A_{h}\right) g\right\|_{0, \Omega} \leqslant C h^{s-2}\|B g\|_{s, \Omega}, \\
\left\|\left(A-A_{h}\right) g\right\|_{1, \Omega}<C h^{s-3}\|B g\|_{s, \Omega}, \\
\left\|\left(B-B_{h}\right) g\right\|_{0, \Omega}<C h^{s-1}\|B g\|_{s, \Omega}, \\
\left\|\left(B-B_{h}\right) g\right\|_{1, \Omega}<C h^{s-1}\|B g\|_{s, \Omega},
\end{gathered}
$$

where $s=\min (r, K+1)$ and $B g \in H^{r}(\Omega)$.

From (7.8c) with $s=3$, we see that $\left\|B-B_{h}\right\|_{\mathfrak{e}\left(L^{2}(\Omega)\right)}<C h^{2}$ and hence that $\lim _{h \rightarrow 0}\left\|B-B_{h}\right\|_{G G}=0$.

We can thus apply Theorems 6.1 and 6.2.

Let $\lambda$ be an eigenvalue of (7.6) and suppose the corresponding eigenfunctions $\psi$ are in $H^{r}(\Omega)$ with $r>3$. Then, using Theorem 6.1 and (7.8), we immediately obtain

$$
\left|\lambda-\lambda_{j h}\right|<C h^{2 s-4}
$$


where $s=\min (r, K+1)$. Note that since $B$ is selfadjoint we obtain estimates for each $\lambda-\lambda_{j h}$ instead of for $\lambda-\tilde{\lambda}_{h}$.

We now turn to eigenfunction estimates. For the sake of simplicity, we assume $\lambda$ has geometric multiplicity 1 . From Theorem 6.2 we obtain

$$
\left\|\psi-\psi_{h}\right\|_{0, \Omega} \leqslant C h^{s-1}
$$

and from Theorem 6.3 we obtain

$$
\left\|u-u_{h}\right\|_{1, \Omega} \leqslant C h^{s-3},
$$

where $s=\min (r, K+1)$ provided $\psi \in H^{r}(\Omega)$. In (7.10), $\psi$ and $\psi_{h}$ are normalized with respect to $\|\cdot\|_{0, \Omega}$, and in (7.11) $u$ and $u_{h}$ are normalized with respect to $\|\cdot\|_{1, \Omega}$. We note that we must check that $\lim _{h \rightarrow 0}\left\|\tau-\tau_{h}\right\|_{X \times W, X \times W}=0$ in order to use Theorem 6.3. This follows from $(7.8 \mathrm{~b})$ and (7.8c) and the regularity estimate $\|B g\|_{3+\varepsilon, \Omega} \leqslant C\|g\|_{0, \Omega}$ for some $\varepsilon>0$.

These techniques can also be applied to the mixed method of Herrmann-Miyoshi [20], [21], [29]. The analysis is essentially the same as that above and we would obtain estimate (7.9) for this method. We note that our analysis of these methods does not yield results when $K=1$. For the case $K=1$ see Ishihara [22], [23].

(7.9) yields an improvement over the estimates in Canuto [9] in the case when the eigenfunctions have low regularity. If, for example, $\psi \in H^{3.5}(\Omega)$ and $K=3$, then (7.9) yields the estimates $\left|\lambda-\lambda_{j h}\right| \leqslant C h^{3}$, whereas the estimates in [9] yield $\left|\lambda-\lambda_{j h}\right|$ $<$ Ch.

Part of the results in this subsection are contained in [38].

(ii) Herrmann-Johnson Method. We consider here a further mixed method for the approximation of (7.6), which has been introduced in [8].

Let $K>1$ and let $\left\{\tau_{h}\right\}$ be a family of triangulations satisfying the minimal angle condition. Given $T \in \tau_{h}$ and a function $\mathrm{v}=\left(v_{i j}\right)$ with $v_{i j} \in H^{1}(T), 1<i, j<2$, and $v_{12}=v_{21}$, we define

$$
M_{\nu}(\mathrm{v})=\sum_{i, j=1}^{2} v_{i j} \nu_{j} \nu_{i} \text { and } M_{v r}(\mathrm{v})=\sum_{i, j=1}^{2} v_{i j} \nu_{j} \tau_{i},
$$

where $\nu=\left(\nu_{1}, \nu_{2}\right)$ is the unit outward normal and $\tau=\left(\tau_{1}, \tau_{2}\right)=\left(\nu_{2},-\nu_{1}\right)$ is the unit tangent along $\partial T$. Let

$$
\begin{aligned}
X=X(h)=\left\{\begin{array}{l}
\mathrm{v}=\left(v_{i j}\right): v_{i j} \in L^{2}(\Omega), v_{12}=v_{21}, v_{i j / \tau} \in H^{1}(T), \\
\left.\forall T \in \tau_{h}, \text { and } M_{\nu}(v) \text { is continuous across interelement boundaries }\right\}
\end{array}\right.
\end{aligned}
$$

with

$$
\|\mathbf{v}\|_{X}^{2}=\sum_{i, j=1}^{2} \sum_{T \in \tau_{h}}\left\|v_{i j}\right\|_{1, T}^{2}
$$

and

$$
W=W_{0}^{1, p}(\Omega), \quad \text { where } p \text { is some number larger than } 2 .
$$

The mixed method we study here is based on the following variational formulation of (7.6): 
Seek $\lambda,(\mathbf{u}, \psi) \in X \times W$ satisfying

$$
\left\{\begin{aligned}
\sum_{i, j=1}^{2} \int_{\Omega} u_{i j} \bar{v}_{i j} d x+\sum_{T \in \tau_{h}}\left\{\sum_{i, j=1}^{2} \int_{T} \frac{\partial \bar{v}_{i j}}{\partial x_{j}} \frac{\partial \psi}{\partial x_{i}} d x-\int_{\partial T} \overline{M_{m}(\mathrm{v})} \frac{\partial \psi}{\partial \tau} d s\right\}=0 & \forall \mathbf{v} \in X \\
\sum_{T \in \tau_{h}}\left\{\sum_{i, j=1}^{2} \frac{\partial u_{i j}}{\partial x_{j}} \frac{\partial \bar{\phi}}{\partial x_{i}} d x-\int_{\partial T} M_{\nu}(u) \frac{\partial \bar{\phi}}{\partial \tau} d s\right\}=-\lambda \int_{\Omega} \psi \bar{\phi} d x, & \forall \phi \in W
\end{aligned}\right.
$$

If $\lambda, \psi$ is an eigenpair of (7.6), then $\lambda,\left(\left(\partial^{2} \psi / \partial x_{i} \partial x_{j}\right), \psi\right)$ is an eigenpair of (7.12), and if $\lambda,\left(\left(u_{i j}\right), \psi\right)$ is an eigenpair of (7.12), then $\lambda, \psi$ is an eigenpair of (7.6) and $u_{i j}=\partial^{2} \psi / \partial x_{i} \partial x_{j}$. (7.12) is a problem of type (Q2) with $X$ and $W$ as above and

$$
\begin{aligned}
& a(\mathbf{u}, \mathbf{v})=\sum_{i, j=1}^{2} \int_{\Omega} u_{i j} \bar{v}_{i j} d x \\
& b(\mathbf{u}, \phi)=\sum_{T \in \tau_{h}}\left\{\sum_{i, j=1}^{2} \int_{T} \frac{\partial u_{i j}}{\partial x_{j}} \frac{\partial \bar{\phi}}{\partial x_{i}} d x-\int_{\partial T} M_{m}(\mathbf{u}) \frac{\partial \bar{\phi}}{\partial \tau} d s\right\} .
\end{aligned}
$$

We consider problem $(\mathrm{Q} 2)_{h}$ with

$$
X_{h}=\left\{\mathrm{v} \in X: v_{i j / T} \in P_{K-1}, \forall T \in \tau_{h}\right\}
$$

and

$$
W_{h}=\left\{\phi \in C^{0}(\bar{\Omega}): \phi_{/ T} \in P_{K}, \forall T \in \tau_{h}, \phi=0 \text { on } \partial \Omega\right\} .
$$

With this choice we have the method of Herrmann-Johnson [20], [21], [25] in case $K=1$ (for $K>1$, see [8]) and we obtain approximations to $\lambda, \partial^{2} \psi / \partial x_{i} \partial x_{j}$ and $\psi$. Our problem fits into the framework of Section 6 with $G=L^{2}(\Omega)$ and $H=$ $\left(L^{2}(\Omega)\right)^{3}$ (cf. remark at the end of Section 6). We can now apply Theorem 6.4 if we remark that the operator $\Pi_{h}$ exists and that $V_{h} \subset V$.

From the results of Subsection 3c in [15], we have

$$
\begin{gathered}
\left\|\left(A-A_{h}\right) f\right\|_{0, \Omega}<C h^{s-2}\|B f\|_{s, \Omega}, \quad 3<s<K+2, \\
\left\|\left(B-B_{h}\right) f\right\|_{0, \Omega}<\left\{\begin{array}{l}
C h^{s-1}\|B f\|_{s, \Omega}, \quad 3<s<K+2, \text { if } K>2, \\
C h^{2}\|B f\|_{4, \Omega}, \quad \text { if } K=1,
\end{array}\right. \\
\inf _{\eta \in W_{h}}\left|b\left(A f-\Pi_{h} A f, B g-\eta\right)\right|<C h^{t+s-4}\|A f\|_{s-2, \Omega}\|B f\|_{t, \Omega,} \\
3<s<K+2,2<t<K+1 .
\end{gathered}
$$

Suppose $\lambda$ is an eigenvalue of (7.12) and suppose the corresponding eigenfunctions $\psi$ are in $H^{\prime}(\Omega)$. Now Theorem 6.4, together with (7.13), yields

$$
\left|\lambda-\hat{\lambda}_{h}\right|<C h^{s+t-4}, \quad s=\min (K+2, r), \quad t=\min (K+1, r) .
$$

This result improves on the result in Canuto [9] if $r<K+2$.

c. In this subsection we discuss a hybrid method for the approximation of the eigenvalues of 2 nd order problems. The related approximation for source problems was studied by Raviart-Thomas [41] and Thomas [44]. As in Subsection 7a, we 
consider the Dirichlet eigenvalue problem

$$
\begin{cases}-\Delta u=\lambda u & \text { in } \Omega, \\ u=0 & \text { on } \Gamma=\partial \Omega,\end{cases}
$$

where $\Omega$ is a convex polygon in $\mathbf{R}^{2}$.

For spaces we choose $H=L^{2}(\Omega), X=\Pi_{T \in \tau_{h}} H^{1}(T)$ with

$$
\begin{gathered}
\|u\|_{X}=\left(\sum_{T \in \tau_{h}}\|u\|_{1, T}^{2}\right)^{1 / 2}, \\
W=\left\{\mu \in \prod_{T \in \tau_{h}} H^{-1 / 2}(\partial T): \text { there exists a function } q \in H(\operatorname{div}, \Omega)\right.
\end{gathered}
$$

$$
\text { such that } \left.q \cdot \nu=\mu \text { on } \partial T, T \in \tau_{h}\right\}
$$

with $\|\mu\|_{w}=\left(\Sigma_{T \in \tau_{h}}\|\mu\|_{-1 / 2, \partial T}^{2}\right)^{1 / 2}$, where

$$
\begin{gathered}
\|\mu\|_{-1 / 2, \partial T}=\inf _{\substack{q \in H(\operatorname{div}, \Omega) \\
q \cdot \nu=\mu \text { on } \partial T}}\|q\|_{H(\operatorname{div}, T)}, \\
\|q\|_{H(\operatorname{div}, T)}=\left(\|q\|_{0, T}^{2}+h_{T}^{2}\|\operatorname{div} q\|_{0, T}^{2}\right)^{1 / 2} .
\end{gathered}
$$

For sesquilinear forms we choose

$$
a(u, v)=\sum_{T \in \tau_{h}} \int_{T} \nabla u \bar{\nabla} v d x \text { and } b(u, \mu)=-\sum_{T \in \tau_{h}} \int_{\partial T} u \bar{\mu} d s,
$$

where the integral over $\partial T$ expresses the duality between $H^{1 / 2}(\partial T)$ and $H^{-1 / 2}(\partial T)$.

We then consider the following formulation of (7.15):

Seek $\lambda,(u, p) \in X \times W$ satisfying

$$
\begin{cases}a(u, v)+\overline{b(v, p)}=\lambda \int_{\Omega} u \bar{v}, & \forall v \in X, \\ b(u, q)=0, & \forall q \in W .\end{cases}
$$

If $\lambda, u$ is an eigenpair of (7.15) and $p=\partial u / \partial \nu$ on $\partial T$ for all $T \in \tau_{h}$, then $\lambda,(u, p)$ is an eigenpair of (7.16), and if $\lambda,(u, p)$ is an eigenpair of (7.16), then $\lambda, u$ is an eigenpair of (7.15) and $p=\partial u / \partial \nu$ on $\partial T$ for all $T \in \tau_{h}$. (7.16) is an eigenvalue problem of type (Q1) with $X, W, H, a$, and $b$ chosen as above and $r(u, v)=$ $\int_{\Omega} u \bar{v} d x$.

We next describe the finite-dimensional approximating spaces that we will use. Let $K \geqslant 1$ be an odd integer. For $X_{h}$ we choose $\Pi_{T \in \tau_{h}} P_{K}(T)$ where $P_{K}(T)$ denotes the space of functions defined on $T$ which are polynomials of degree less than or equal to $K$. For $W_{h}$ we choose

$$
W_{h}=\left\{\mu \in W: \mu_{/ \partial T} \in S_{K-1}(\partial T)\right\},
$$

where $S_{K-1}(\partial T)$ is the space of all functions defined on $\partial T$ whose restrictions to any side $T^{\prime} \subset \partial T$ are polynomials of degree less than or equal to $K-1$. For a more complete treatment of these spaces as well as a description of families of approximating spaces indexed by even $K$ we refer to [41], [44].

We now recall the basic estimates for the errors $\left(A-A_{h}\right) g$ and $\left(B-B_{h}\right) g$ which are proved in Raviart-Thomas [41] and Thomas [44]: 


$$
\begin{gathered}
\left\|\left(A-A_{h}\right)_{g}\right\|_{X}+\left\|\left(B-B_{h}\right) g\right\|_{W}<C h^{\prime}\|A g\|_{l+1, \Omega}, \\
\left\|\left(A-A_{h}\right) g\right\|_{0, \Omega}<C h^{l+1}\|A g\|_{l+1, \Omega},
\end{gathered}
$$

for $l=1, \ldots, K$, provided $A g \in H^{l+1}(\Omega)$.

We have $V=H^{2}(\Omega) \cap H_{0}^{1}(\Omega)$; our problem fits into the framework of Section 5 (cf. remark following Theorem 5.2) and we can thus estimate the eigenvalue errors with Theorem 5.1. $A$ is selfadjoint in this example. Let $\lambda$ be an eigenvalue of (7.15) and suppose the corresponding eigenfunctions $u$ are in $H^{l+1}(\Omega)$ with $1<l<K$. Then combining Theorem 5.1 and Estimate (7.17) we have

$$
\left|\lambda-\lambda_{j h}\right|<C h^{2 l} \text {. }
$$

We now consider eigenfunction errors. We assume $\lambda$ has geometric multiplicity 1 for the sake of simplicity. From Theorem 5.2 and (7.18) we get

$$
\left\|u-u_{h}\right\|_{0, \Omega}<C h^{l+1}
$$

provided $u \in H^{l+1}(\Omega)$. Here $u$ and $u_{h}$ are normalized with respect to $\|\cdot\|_{0, \Omega}$.

d. In this subsection we consider the approximation of an eigenvalue problem associated with the Stokes problem by a method developed in Girault-Raviart [18].

Let $\Omega$ be a convex polygon in $\mathbf{R}^{2}$ and consider the eigenvalue problem

Find $\lambda, \vec{u}$ and $p$ satisfying

$$
\begin{cases}-\Delta \vec{u}+\overrightarrow{\operatorname{grad}} p=\lambda \vec{u} & \text { in } \Omega, \\ \operatorname{div} \vec{u}=0 & \text { in } \Omega, \\ \vec{u}=0 & \text { on } \Gamma=\partial \Omega .\end{cases}
$$

If we introduce the stream function $\psi(\vec{u}=\overrightarrow{c u r l} \psi)$, this problem can be formulated as:

Find $\lambda, \psi$ satisfying

$$
\begin{cases}-\Delta^{2} \psi=\lambda \Delta \psi & \text { in } \Omega, \\ \psi=\frac{\partial \psi}{\partial \nu}=0 & \text { on } \Gamma .\end{cases}
$$

We then consider the following mixed formulation of (7.22) (introduced in [18] for source problem):

Find $\lambda,(u, \mu) \in X \times W$ satisfying

$$
\begin{cases}a(u, v)+\overline{b(v, \mu)}=\lambda r(u, v), & \forall v \in X, \\ b(u, q)=0, & \forall q \in W,\end{cases}
$$

where

$$
\begin{aligned}
X & =H_{0}^{1}(\Omega) \times L^{2}(\Omega), \\
W & =H^{1}(\Omega), \\
a(u, v) & =\int_{\Omega} \omega \bar{\theta} d x \quad \text { for } u=(\psi, \omega), v=(\phi, \theta) \in X, \\
b(u, q) & =\int_{\Omega}(\overrightarrow{\operatorname{curl}} \bar{q} \overrightarrow{\operatorname{curl}} \psi-\bar{q} \omega) d x \quad \text { for } u \in X, q \in W, \\
r(u, v) & =\int_{\Omega} \overrightarrow{\operatorname{curl}} \psi \overrightarrow{\operatorname{curl}} \bar{\phi} d x \quad \text { for } u, v \in X,
\end{aligned}
$$

where $\overrightarrow{\operatorname{curl}} \phi=\left(-\partial \phi / \partial x_{2}, \partial \phi / \partial x_{1}\right)$. 
If $\lambda, \psi$ is an eigenpair of (7.22) and $\omega=\mu=-\Delta \psi$, then $\lambda,(u=(\psi, \omega), \mu)$ is an eigenpair of (7.23), and if $\lambda,(u=(\psi, \omega), \mu)$ is an eigenpair of (7.23) then $\lambda, \psi$ is an eigenpair of (7.22) and $\omega=\mu=-\Delta \psi$. The eigenvalue problem (7.23) is of type (Q1). Note that assumption (3.13) is not satisfied here.

Next we consider the approximation method introduced in Girault-Raviart [18]. We again let $\left\{\tau_{h}\right\}$ be a quasiuniform family of triangulations of $\bar{\Omega}$ that satisfies the minimal angle condition and let

$$
W_{h}=\left\{q \in C^{0}(\bar{\Omega}): q_{/ T} \in P_{K}, \forall T \in \tau_{h}\right\} \text { and } X_{h}=\left(W_{h} \cap H_{0}^{1}(\Omega)\right) \times W_{h} \text {. }
$$

Then we consider the following approximate problem:

Find $\lambda_{h},\left(u_{h}, \mu_{h}\right) \in X_{h} \times W_{h}$ satisfying

$$
\begin{cases}a\left(u_{h}, v_{h}\right)+\overline{b\left(v_{h}, \mu_{h}\right)}=\lambda_{h} r\left(u_{h}, v_{h}\right), & \forall v_{h} \in X_{h}, \\ b\left(u_{h}, q_{h}\right)=0, & \forall q_{h} \in W_{h} .\end{cases}
$$

(7.23) and (7.24) fit into the framework of Section 5 with $X, W, a, b$, and $r$ defined as above and $H=H_{0}^{1}(\Omega) \times L^{2}(\Omega)$. In this case, we have

$$
V=\{v \in X: b(v, q)=0, \forall q \in W\}=\left\{(\phi, \theta): \phi \in H_{0}^{2}(\Omega) \text { and } \theta=-\Delta \phi\right\} \text {, }
$$

and $a(\cdot, \cdot)$ is $V$-elliptic.

We now recall the basic estimates for the error $\left(A-A_{h}\right) g$ of the source problem, which are proved in Girault-Raviart [18].

Set $A g=(\phi, \theta), A_{h} g=\left(\phi_{h}, \theta_{h}\right)$. We have

$$
\begin{gathered}
\left\|\left(A-A_{h}\right) g\right\|_{H} \leqslant C h\|g\|_{H}, \\
\left\|\left(A-A_{h}\right) g\right\|_{X} \leqslant C h^{K-1 / 2}\left\{\|\phi\|_{K+1, \infty, \Omega}+\|\phi\|_{K+3 / 2,2, \Omega}\right\},
\end{gathered}
$$

if $\phi \in W^{K+1, \infty}(\Omega) \cap H^{K+3 / 2}(\Omega)$,

$$
\left\|\phi-\phi_{h}\right\|_{1, \Omega} \leqslant C h^{K}\|\phi\|_{K+1, \Omega}
$$

if $\phi \in H^{K+1}(\Omega)$, provided $K \geqslant 2$.

Let $\lambda$ be an eigenvalue of (7.22) and let $\mathcal{E}$ be the corresponding eigenspace. Then $\lambda^{-1}$ will be an eigenvalue of $A$ with the eigenspace given by $E=\{(\psi,-\Delta \psi)$ : $\psi \in \mathcal{E}\}$. Let $m$ be the multiplicity of $\lambda^{-1}$. From (7.25) we see that

$$
\lim _{h \rightarrow 0}\left\|A-A_{h}\right\|_{H H}=0 \text {. }
$$

Thus, $m$ eigenvalues $\lambda_{1 h}^{-1}, \ldots, \lambda_{m h}^{-1}$ of $A_{h}$ converge to $\lambda^{-1}$. Assume $\mathcal{E} \subset W^{K+1, \infty}(\Omega)$ $\cap H^{K+2}(\Omega)$. We can now estimate the terms on the right side of (5.8).

From (7.26), we have

$$
\left\|\left(A-A_{h}\right)_{/ E}\right\|_{H X}^{2} \leqslant C h^{2 K-1} .
$$

Next, let $f, g \in E$ with $\|f\|_{H}=\|g\|_{H}=1$ and set $A f=(\phi, \theta)$ and $A_{h} f=\left(\phi_{h}, \theta_{h}\right)$. Then, using (7.26) and (7.27), we have

$$
\begin{aligned}
\left|b\left(\left(A-A_{h}\right) f, B g-\eta\right)\right| & =\mid \int_{\Omega} \overrightarrow{\operatorname{curl}}\left(\phi-\phi_{h}\right) \overrightarrow{\operatorname{curl}}(B g-\eta) d x \\
& \leqslant C\left\{\left\|\phi-\phi_{h}\right\|_{1, \Omega}\|B g-\eta\|_{1, \Omega}+\left\|\theta-\theta_{h}\right\|_{0, \Omega}\|B g-\eta\|_{0, \Omega}\right\} \\
& \leqslant C\left\{h^{K}\|B g-\eta\|_{1, \Omega}+h^{K-1 / 2}\|B g-\eta\|_{0, \Omega}\right\} \\
& \leqslant C h^{K-1}\left\{h\|B g-\eta\|_{1, \Omega}+\|B g-\eta\|_{0, \Omega}\right\}
\end{aligned}
$$


for any $\eta \in W_{h}$. Since $B g \in H^{K}(\Omega)$, by standard approximation results we have

$$
\inf _{\eta \in W_{h}}\left|b\left(\left(A-A_{h}\right) f, B g-\eta\right)\right|<C h^{2 K-1} .
$$

Finally, combining (5.8), (7.28), and (7.29), we have

$$
\left|\lambda-\lambda_{j h}\right| \leqslant C h^{2 K-1}, \quad j=1,2, \ldots, m .
$$

e. In this subsection we consider a method introduced by Crouzeix-Raviart [11] for the approximation of the eigenvalues of the Stokes problem (7.21). Let

$$
\begin{gathered}
X=\mathbf{H}_{0}^{1}(\Omega), \quad W=L^{2}(\Omega)_{/ \mathbf{R}}, \\
a(u, v)=\sum_{i, j=1}^{2} \int_{\Omega} \frac{\partial u_{i}}{\partial x_{j}} \frac{\partial v_{i}}{\partial x_{j}} d x \quad \text { for } u, v \in X, \\
b(u, q)=-\int_{\Omega} q \operatorname{div} u d x \quad \text { for } u \in X, q \in W .
\end{gathered}
$$

Then we consider the following formulation of (7.21):

Find $\lambda,(u, p) \in X \times W$ satisfying

$$
\begin{cases}a(u, v)+b(v, p)=\lambda(u, v)_{\mathbf{L}^{2}(\Omega),} & \forall v \in X, \\ b(u, q)=0, & \forall q \in W .\end{cases}
$$

We next consider the finite-dimensional approximating spaces that we will use. Suppose $F_{h} \subset H_{0}^{1}(\Omega)$ and $G_{h} \subset L^{2}(\Omega)$ are given finite-dimensional spaces and $X_{h}=F_{h}^{2}$ and $W_{h}=G_{h / \mathrm{R}}$. Regarding these spaces, we assume

(H1) there is a bounded operator $r_{h}:\left(\mathbf{H}^{2}(\Omega) \cap \mathbf{H}_{0}^{1}(\Omega)\right) \rightarrow W_{h}$ that satisfies

(i) $\int_{\Omega} q \operatorname{div}\left(v-r_{h} v\right) d x=0$ for all $q \in G_{h}$,

(ii) there is a positive integer $K$ such that

$$
\left\|r_{h} v-v\right\|_{\mathbf{H}^{\prime}(\Omega)}<C h^{\prime}\|v\|_{\mathbf{H}^{\prime+1}(\Omega)} \text { for } 1 \leqslant l<K,
$$

and

(H2) the spaces $G_{h}$ contain constants, and if $P_{h}$ is the orthogonal projection of $L^{2}(\Omega)$ onto $G_{h}$, then

$$
\int_{\Omega} q d x=0 \text { implies } \int_{\Omega} P_{h} q d x=0
$$

and $\left\|q-P_{h} q\right\|_{0, \Omega} \leqslant C h^{K}\|q\|_{K, \Omega}, 1<l<K$.

Several examples of families of spaces satisfying $(\mathrm{H} 1)$ and $(\mathrm{H} 2)$ for various values of $l$ are constructed in Crouzeix-Raviart [11].

With $X_{h}$ and $W_{h}$ defined as above, we consider the approximate problem:

Find $\lambda_{h},\left(u_{h}, p_{h}\right) \in X_{h} \times W_{h}$ satisfying

$$
\begin{cases}a\left(u_{h}, v_{h}\right)+b\left(v_{h}, p_{h}\right)=\lambda_{h}\left(u_{h}, v_{h}\right)_{\mathbf{L}^{2}(\Omega)}, & \forall v_{h} \in X_{h}, \\ b\left(u_{h}, q_{h}\right)=0, & \forall q_{h} \in W_{h} .\end{cases}
$$

(7.31) and (7.32) fit into the framework of Section 5 with $X, W, a$, and $b$ defined as above and $H=\mathbf{L}^{2}(\Omega)$. The eigenvalue error that arises in this approximation can now be estimated with the aid of Theorem 5.1. Regarding the associated source and approximate source problems, Crouzeix-Raviart [11] have shown that

$$
\left\|\left(A-A_{h}\right) f\right\|_{X}<C h^{l}\left(\|A f\|_{\mathbf{H}^{\prime+1}(\Omega)}+\|B f\|_{l, \Omega}\right)
$$


and

$$
\left\|\left(B-B_{h}\right) f\right\|_{W} \leqslant C h^{l}\left(\left\|A_{f}\right\|_{\mathbf{H}^{l+1}(\Omega)}+\|B f\|_{l, \Omega)}\right) \text { for } 1<l<K .
$$

Let $\lambda^{-1}$ be an eigenvalue of $A$ with multiplicity $m$. Then $m$ eigenvalues $\lambda_{1 h}^{-1}, \ldots, \lambda_{m h}^{-1}$ of $A_{h}$ converge to $\lambda^{-1}$. Suppose that the associated space $E$ of eigenfunctions satisfies $E \subset \mathbf{H}^{K+1}(\Omega)$ and $B(E) \subset H^{K}(\Omega)$. Then it follows immediately from Theorem 5.1, (7.33), and (7.34) that

$$
\left|\lambda-\lambda_{j h}\right|<C h^{2 l}, \quad 1 \leqslant j \leqslant m,
$$

for $1 \leqslant l \leqslant K$. (7.35) was proved by Osborn [37]. We remark that this method can also be analyzed by means of the results in Section 3 .

C. E. A. Limeil

B.P. 27

94190 Villeneuve, St. Georges, France

Department of Mathematics

University of Maryland

College Park, Maryland 20742

Centre de Mathématiques Appliquées

Ecole Polytechnique

91128 Palaiseau Cedex, France

Analyse Numérique

Université Pierre et Marie Curie

5 Place Jussieu

75230 Paris Cedex 05, France

1. I. BABUŠKA, "Error-bounds for finite element methods," Numer. Math., v. 16, 1971, pp. 322-333.

2. I. BABUŠKA \& A. AZIZ, "Survey lectures on the mathematical foundations of the finite element method" in The Mathematical Foundations of the Finite Element Method with Application to Partial Differential Equations (A. K. Aziz, Ed.), Academic Press, New York, 1973, pp. 5-359.

3. I. BABUŠKA \& J. OsBORN, "Numerical treatment of eigenvalue problems for differential equations with discontinuous coefficients," Math. Comp., v. 32, 1978, pp. 991-1023.

4. J. BRAMBLE \& J. OsBORN, "Rate of convergence estimates for non-selfadjoint eigenvalue approximations," Math. Comp., v. 27, 1973, pp. 525-549.

5. I. Babušsa, J. Osborn \& J. Pitkäranta, "Analysis of mixed methods using mesh dependent norms," Math. Comp., v. 35, 1980, pp. 1039-1062.

6. F. BrezzI, "On the existence, uniqueness and approximation of saddlepoint problems arising from Lagrangian multipliers," R.A.I.R.O., v. 8, R-2, 1974, pp. 129-151.

7. F. Brezzi, "Sur la méthode des éléments finis hybrides pour le problème biharmonique," Numer. Math., v. 24, 1975, pp. 103-131.

8. F. Brezzi \& P. A. Raviart, "Mixed finite element methods for 4th order elliptic equations," Topics in Numerical Analysis III (J. Miller, Ed.), Academic Press, New York, 1978.

9. C. Canuto, "Eigenvalue approximations by mixed methods," R.A.I.R.O. Anal. Numér., v. 12, 1978, pp. 27-50.

10. P. Ciarlet \& P. A. Raviart, "A mixed finite element method for the biharmonic equation," Symposium on Mathematical Aspects of Finite Elements in Partial Differential Equations (C. de Boor, Ed.), Academic Press, New York, 1974, pp. 125-143.

11. M. Crouzeix \& P. A. Raviart, "Conforming and nonconforming finite element methods for solving the stationary Stokes equations. I," R.A.I.R.O., v. 7, R-3, 1973, pp. 33-76.

12. J. Descloux, N. NASSIF \& J. RAPPAZ, "On spectral approximation, Part 2: Error estimates for the Galerkin method," R.A.I.R.O. Anal. Numér., v. 12, 1978, pp. 113-119.

13. J. Descloux, N. Nassif \& J. Rappaz, Various Results on Spectral Approximation, Rapport du Dept. de Math. de l'Ecole Polytechnique Fédérale de Lausanne, Suisse, 1977. 
14. R. FALK, "Approximation of the biharmonic equation by a mixed finite element method," SIAM J. Numer. Anal., v. 15, 1978, pp. 556-567.

15. R. FAlk \& J. OsborN, "Error estimates for mixed methods," R.A.I.R.O. Anal. Numér., v. 14, 1980, pp. 249, 277.

16. G. Fix, "Eigenvalue approximation by the finite element method," Adv. in Math., v. 10, 1973, pp. 300-316.

17. M. Fortin, "Analysis of the convergence of mixed finite element methods," R.A.I.R.O., v. 11, 1977, pp. 341-354.

18. V. Girault \& P. A. Raviart, "An analysis of a mixed finite element method for the NavierStokes equations,” Numer. Math., v. 33, 1979, pp. 235-271.

19. R. Glowinski, “Approximations externes par éléments finis de Lagrange d'ordre un et deux, du problème de Dirichlet pour l'opérateur biharmonique, Méthodes itératives de résolutions des problèmes approchés," in Topics in Numerical Analysis (J. J. H. Miller, Ed.), Academic Press, New York, 1973, pp. 123-171.

20. L. Herrmann, "Finite element bending analysis for plates," J. Eng. Mech., Div. ASCE EM5, v. 93, 1967, pp. 49-83.

21. L. Herrmann, "A bending analysis for plates," Proc. Conf. on Matrix Methods in Structural Mechanics, AFFDL-TR-66-88, pp. 577-604.

22. K. IshinaRA, "Convergence of the finite element method applied to the eigenvalue problem $\Delta u+\lambda u=0$," Publ. Res. Inst. Math. Sci., v. 13, 1977, pp. 48-60.

23. K. Ishiнara, "The buckling of plates by the mixed finite element method," Mem. Numer. Math., v. 5, 1978, pp. 73-82.

24. K. IsHihARA, "A mixed finite element method for the biharmonic eigenvalue problem of plate bending," Publ. Res. Inst. Math. Sci., v. 14, 1978, pp. 399-414.

25. C. Johnson, "On the convergence of a mixed finite element method for plate bending problems," Numer. Math., v. 21, 1973, pp. 43-62.

26. W. Kolata, “Approximation of variationally posed eigenvalue problems," Numer. Math., v. 29, 1978, pp. 159-171.

27. B. Mercier, "Numerical solution of the biharmonic problems by mixed finite elements of class C 0," Boll. Un. Mat. Ital., v. 10, 1974, pp. 133-149.

28. B. MERCIER \& J. RAPPAZ, Eigenvalue approximation via non-conforming and hybrid finite element methods, Rapport du Centre de Mathématiques Appliquées, Ecole Polytechnique, Palaiseau, France, 1978.

29. T. MrYoshi, “A finite element method for the solution of fourth order partial differential equations,” Kumamoto J. Sci. (Math.), v. 9, 1973, pp. 87-116.

30. S. Nemat-NASSER, "General variational methods for elastic waves in composites," J. Elasticity, v. 2, 1972, pp. 73-90.

31. S. Nemat-Nasser, "Harmonic waves in layered composites," J. Appl. Mech., v. 39, 1972, pp. 850-852.

32. S. Nemat-NASSER, General Variational Principles in Nonlinear and Linear Elasticity with Applications. Mechanics Today 1, Pergamon Press, New York, 1974, pp. 214-261.

33. T. ODEN, "Some contributions to the mathematical theory of mixed finite element approximations," in Theory and Practice in Finite Element Structural Analysis, Univ. of Tokyo Press, Tokyo, 1973, pp. 3-23.

34. T. ODEN, "Some contributions to the mathematical theory of mixed finite element approximations," in Theory and Practice in Finite Element Structural Analysis, Univ. of Tokyo Press, Tokyo, 1973, pp. 3-23.

35. T. ODEN \& J. REDDY, “On mixed finite element approximations," SIAM J. Numer. Anal., v. 13, 1976, pp. 393-404.

36. J. OsBoRN, "Spectral approximation for compact operators," Math. Comp., v. 29, 1975, pp. 712-725.

37. J. OsBorN, “Approximation of the eigenvalues of a non-selfadjoint operator arising in the study of the stability of stationary solutions of the Navier-Stokes equations," SIAM J. Numer. Anal., v. 13, 1976, pp. 185- 197.

38. J. Osborn, Advances in Computer Methods for Partial Differential Equations III, Proc. Third IMACS Internat. Sympos. on Computer Methods for Partial Differential Equations held at Lehigh University, Bethlehem, Pennsylvania, June 1979, (R. Vichnevetsky, R. Stepleman, Eds.).

39. R. RANNAChER, "On nonconforming and mixed finite element methods for plate bending problems-the linear case." (Preprint.) 
40. P. A. Raviart \& J. M. Thomas, A Mixed Finite Element Method for 2nd Order Elliptic Problems, Lecture Notes in Math., Vol. 606, Springer-Verlag, Berlin and New York, 1977, pp. 292-315.

41. P. A. Raviart \& J. M. Thomas, "Primal hybrid finite element method for 2 nd order elliptic problems," Math. Comp., v. 31, 1977, pp. 391-413.

42. R. Scholz, Approximation von Sattelpunkten mit Finiten Elementen, Tagungsband, Bonn. Math. Schr., Vol. 89, 1976, pp. 53-66.

43. R. SCholz, "A mixed method for 4th order problems using linear finite elements," R.A.I.R.O. Numér. Anal., v. 12 1978, pp. 85-90.

44. J. M. Thомаs, Sur l'Analyse Numérique des Méthodes d'Eléments Finis Hybrides et Mixtes, Thesis, Univ. P \& M Curie, Paris, 1977.

45. J. Wilkinson, The Algebraic Eigenvalue Problem, Oxford Univ. Press, 1965. 